\title{
Observation evidence for the entropy switch model of substorm onset
}

\author{
YunXiang Song, and ChuXin Chen* \\ CAS Key Laboratory of Geospace Environment, Department of Geophysics and Planetary Sciences, School of Earth and Space Sciences, University of Science \\ and Technology of China, Hefei 230026, China
}

\section{Key Points:}

- Interchange or ballooning instability in the near-Earth tail is the dominant cause of substorm onset.

- The entropy switch model of substorm onset is supported by observations.

- Both a case study and a statistical study with THEMIS data were used in supporting this conclusion.

Citation: Song, Y. X., and Chen, C. X. (2022). Observation evidence for the entropy switch model of substorm onset. Earth Planet. Phys., 6(2), 161-176. http://doi.org/10.26464/epp2022020

\begin{abstract}
The cause of substorm onset is not yet understood. Chen CX (2016) proposed an entropy switch model, in which substorm onset results from the development of interchange instability. In this study, we sought observational evidence for this model by using Time History of Events and Macroscale Interactions during Substorms (THEMIS) data. We examined two events, one with and the other without a streamer before substorm onset. In contrast to the stable magnetosphere, where the total magnetic field strength is a decreasing function and entropy is an increasing function of the downtail distance, in both events the total magnetic field strength and entropy were reversed before substorm onset. After onset, the total magnetic field strength, entropy, and other plasma quantities fluctuated. In addition, a statistical study was performed. By confining the events with THEMIS satellites located in the downtail region between $\sim 8$ and $\sim 12$ Earth radii, and 3 hours before and after midnight, we found the occurrence rate of the total magnetic field strength reversal to be $69 \%$ and the occurrence rate of entropy reversal to be $77 \%$ of the total 205 events.
\end{abstract}

Keywords: substorm onset; entropy switch model; interchange or ballooning instability; Time History of Events and Macroscale Interactions during Substorms (THEMIS) data

\section{Introduction}

Substorms are global reconfigurations of the magnetosphere involving the storage of solar wind energy in the Earth's magnetotail and its abrupt conversion to particle heating and kinetic energy. They are associated with a dynamic reconfiguration of the magnetic field and highly variable plasma distributions in the magnetosphere.

Substorm onset triggers, a controversial topic, have been studied for many years. The sequence of events leading to substorm onset has remained unsolved. There are two main schools of thought on the cause of substorms. The first "outside-in" emphasizes the role of magnetic reconnection in the midtail region $\left(X_{\mathrm{gsm}}\right.$ $\approx-15$ to $-30 R_{\mathrm{E}}$, where $R_{\mathrm{E}}$ is the Earth's radius; Hones, 1977). A substorm onset follows when a streamer (the accompanying aurora signature of a flow burst in the magnetosphere) approaches the thin arc (Henderson et al., 1998; Sergeev et al., 1999; Nishimura et al., 2010, 2011). The second "inside-out" focuses on the role of plasma instabilities in the near-Earth magnetotail re-

Correspondence to: C. X. Chen, chuxin@ustc.edu.cn

Received 11 OCT 2021; Accepted 17 JAN 2022.

Accepted article online 28 JAN 2022.

C 2022 by Earth and Planetary Physics. gion ( $X_{\mathrm{gsm}} \approx-6$ to $-15 R_{\mathrm{E}} ;$ Lui, 1991). Substorm onsets take place without a streamer. The two opposing models both have observational evidence.

Chen CX (2016) proposed a scenario for a substorm onset trigger. In this model, substorm onset is regarded as a switch on the sequence of transport from decreasing entropy to increasing entropy. Entropy $p V^{5 / 3}$ (where $p$ is the thermal pressure and $V$ is the volume of one unit magnetic flux tube) is a critical parameter in determining the motion of a flux tube and its rest location in the magnetosphere. In a stable magnetosphere, entropy tends to increase tailward (Wolf et al., 2009).

Pontius and Wolf (1990) and Chen CX and Wolf $(1993,1999)$ pointed out that a bubble (magnetic flux tube with entropy lower than its neighbors) would move earthward through the plasma sheet and come to rest where its entropy matched those of its neighbors. Toffoletto et al. (2000) and Wolf et al. (2002) inferred on theoretical grounds that flux tubes with relatively lower entropy would be injected into the inner magnetosphere (Chen CX and Wang CP, 2019; Chen CX, 2021) during the substorm growth phase. Observations reported by Lyons et al. (2003) seemed to support this view.

As Chen CX (2016) pointed out, in the growth phase of a sub- 
storm, the successive bubbles are in an entropy-decreasing order because the magnetotail becomes more stretched and the location of the reconnection site may shift earthward. In the later growth phase, an early-arriving high-entropy bubble will block a later-arriving low-entropy bubble tailward, forming an unstable domain. The development of the interchange instability leads to the collapse of the stretched plasma sheet, and the substorm expansion phase follows.

The entropy switch model (Chen CX, 2016) is a more general model of substorm onset that combines the inside-out and outside-in models. For the inside-out situation, the unstable domain is formed by a sudden decrease in entropy flow with no streamer before substorm onset, whereas for the outside-in situation, the unstable domain is formed by a lower entropy bubble with an accompanying auroral streamer.

In this study, we sought to check the validity of the entropy switch model by using observational data. Entropy is a global quantity, which involves an integral of the volume (of one unit magnetic flux tube) along the length of a flux tube. Although entropy is difficult to measure directly, the magnetic field and plasma pressure inside the unstable domain are observable. When two successive bubbles are relatively stationary in relation to each other, as shown in Figure 1, the lengths of their flux tubes are almost the same because the unstable domain is located at the region around -8 to $-12 R_{\mathrm{E}}$, and the tailward dimension of the unstable domain is less than $1 R_{\mathrm{E}}$ (Chen CX, 2016). Thus, entropy is proportional to the measured pressure and inversely proportional to $5 / 3$ power of the measured magnetic field. We used individual events and statistical analyses to ascertain whether a substorm onset was

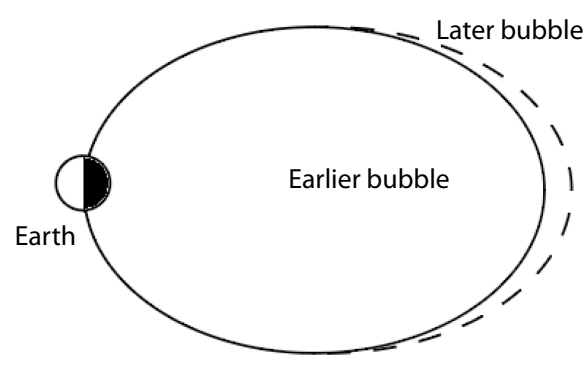

Figure 1. Diagram of an unstable domain.

triggered by the interchange or by ballooning instability.

The remainder of this paper is arranged as follows. The data set from Time History of Events and Macroscale Interactions during Substorms (THEMIS; Angelopoulos, 2008) observations is introduced in Section 2. In Section 3, we examine two events, one with a streamer before substorm onset and the other without a preceding streamer. In Section 4, we report the results of a statistical study using 205 events. The summary and discussion are given in Section 5 .

\section{Data Set}

According to a discussion of the interchange instability in Chen CX $(2013,2016)$, we know that the unstable domain should be located in the dipolar region near the Earth at about 8 to $12 R_{\mathrm{E}}$ in the magnetotail, and the tailward extension of the unstable domain should be less than $1 R_{\mathrm{E}}$. We utilized observations from the THEMIS spacecraft (Angelopoulos, 2008).

The quantities of interest were the magnetic field, particle pressure, ion density, and ion velocity. The magnetic field data were taken from the fluxgate magnetometer (Auster et al., 2008). Data from the particle detectors of the electrostatic analyzer (ESA; McFadden et al., 2008) were used for particle pressure, ion density, and ion velocity. In addition, all-sky-camera images (Mende et al., 2008) were used to identify whether an event had a streamer.

The events were chosen based on the following criteria. During the substorm onset, the THEMIS satellites were located in the radius distance between $\sim 8$ and $\sim 12 R_{\mathrm{E}}$ and at the local time of 3 hours before and after the midnight. The time range of data was chosen as 2 minutes before and after the substorm onset.

The entropy $S\left(p V^{5 / 3}\right)$ involves an integral of the volume $V$ (of one unit magnetic flux tube) along the length of a flux tube. The volume $V$ can be represented as

$$
V=\int \frac{\mathrm{d} s}{B}
$$

where $B$ is the magnetic field and $s$ is the distance along the flux tube. Thus, the entropy $S$ can be expressed as

$$
S=p\left(\int \frac{\mathrm{d} s}{B}\right)^{5 / 3} \text {. }
$$

Because the distance along the flux tube is difficult to measure directly, we estimated an entropy-related quantity $S R$ instead. The $S R$ can be expressed as

$$
S R=p\left(\frac{1}{B}\right)^{5 / 3} .
$$

To compare the entropy $S$ of two neighboring flux tubes, we compared the entropy-related quantity $S R$ instead so that the difference in the lengths of the neighboring flux tubes could be ignored, as indicated in Figure 1.

\section{Case Study}

\subsection{The Event on February 28, 2009, Without a Streamer} At 02:24 UT on February 28, 2009, a substorm onset event was detected in the near-Earth region by THEMIS THA and THE (Lui, 2011). THEMIS THA and THE were at $(X, Y, Z)_{\mathrm{GSM}}=(-8.15,2.41$, $-2.25) R_{\mathrm{E}}$ and $(-8.17,2.51,-1.56) R_{\mathrm{E}}$ at 02:24 UT. THEMIS THA and THE satellite footprints as projected on the Earth's ionosphere and their orbits in the $X Y, X Z, Y Z$ plane of the geocentric solar magnetospheric (GSM) coordinate system are shown in Figure 2. Although THA was closer to Earth than THE in the $X$ direction of the GSM coordinates, THA had a larger radius distance than THE, as we can see from Figures $2 \mathrm{c}$ and $2 \mathrm{~d}$ as well as in the labels under the $x$-axis of Figure 3. Therefore, the footprints of THA on the ionosphere were located more poleward than those of THE when projected from the equatorial plane, as shown in Figure 2a. Both satellites are located near midnight. In addition, the two spacecraft were separated by $4.5^{\circ}$ in azimuth; thus, the two satellites were roughly in line with the Earth. 

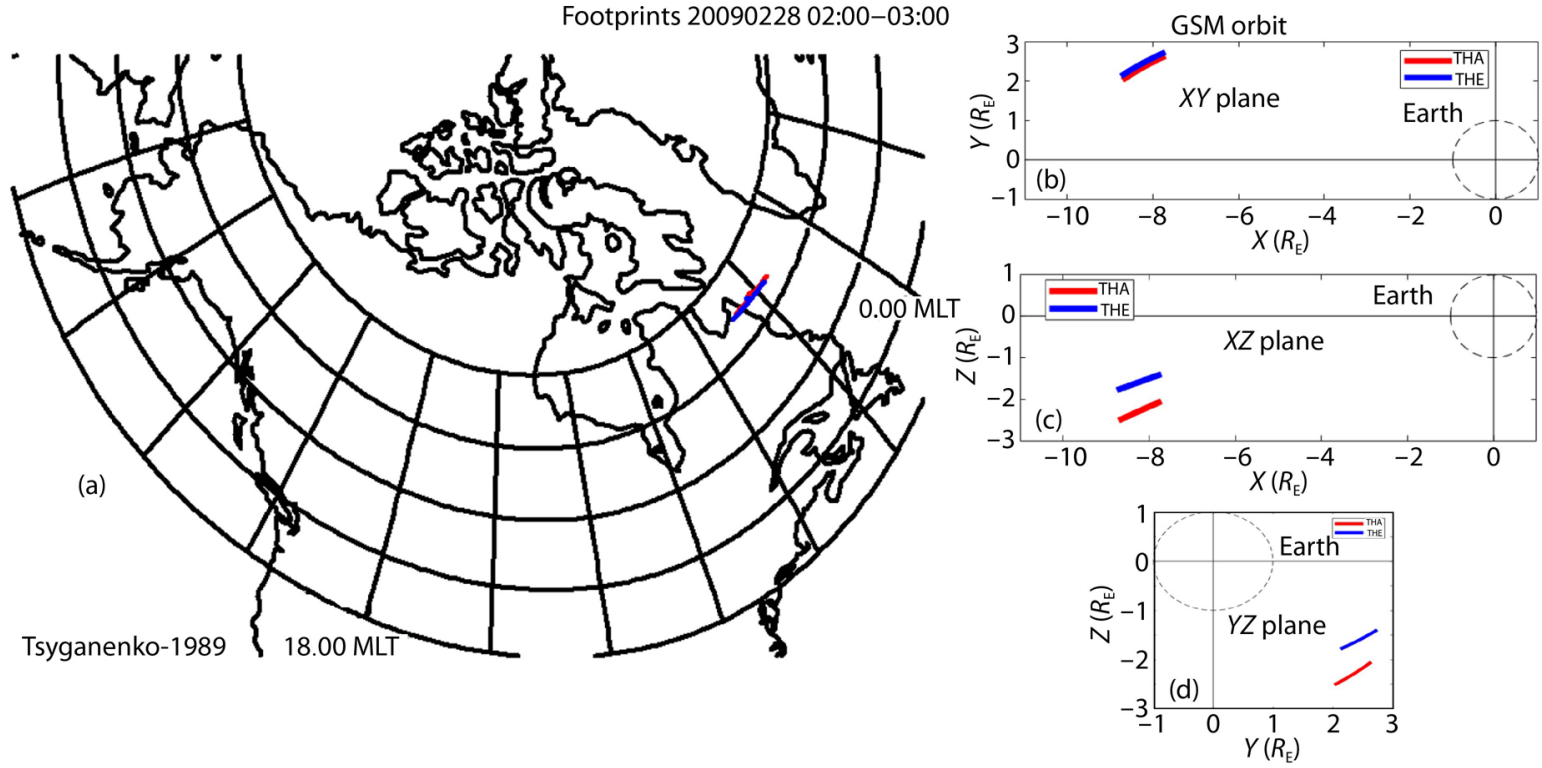

Figure 2. THEMIS THA (red) and THE (blue) satellite footprints as projected on the Earth's ionosphere (a) on February 28, 2009, from 02:00 to 03:00 UT, and their orbits in GSM coordinates $(b-d)$. The footprints were individually projected along the local magnetic field line as described by the model of Tsyganenko (1989). The 0.00 magnetic local time (MLT) in (a) indicates midnight. The orbits of satellites THA and THE in the $X Y, X Z$, YZ plane of the GSM coordinate system are shown in (b), (c), and (d). The red line indicates the orbit of THA, and the blue line is the orbit of THE.

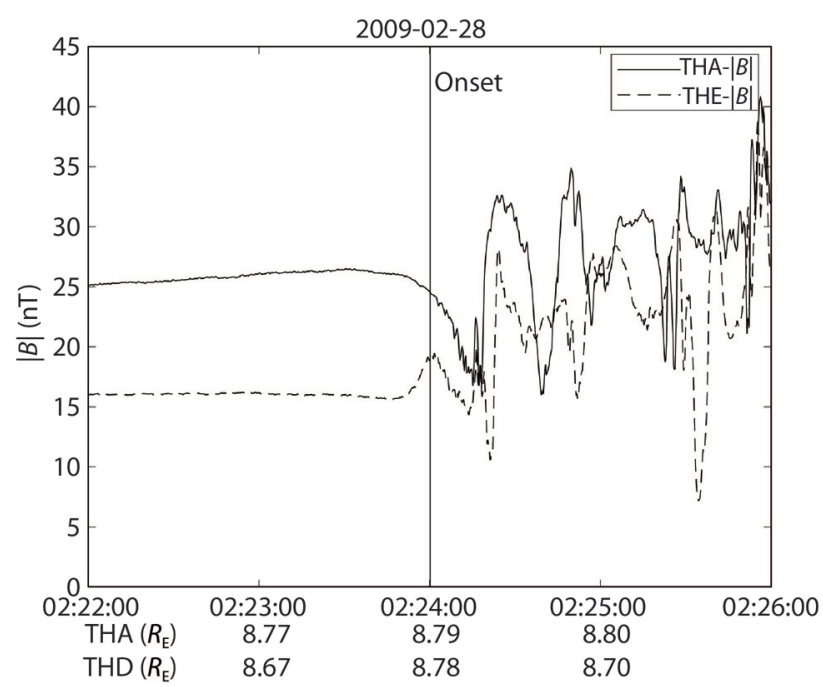

Figure 3. The total magnetic field strengths from THEMIS THA and THE in GSM coordinates around 02:24 UT on February 28, 2009. The time range is 2 minutes before and after the substorm onset. The solid line is the total magnetic field strength of THA, and the dashed line is the total magnetic field strength of THE. The solid black vertical line indicates the substorm onset time at 02:24 UT. The onset time is determined by the auroral electrojet $(A E)$ index, with a resolution of one minute. The Earth radius distances of the two spacecraft are shown under the $x$-axis.

Figure 3 shows the total magnetic field strength of THA and THE for this event. The time range is 2 minutes before and after the substorm onset, with a resolution of $0.25 \mathrm{~s}$. Before the substorm onset, the total magnetic field strength has a typical value of 26 $\mathrm{nT}$ at the THA location and $16 \mathrm{nT}$ at the THE location. In a stable dipole magnetic field, the total magnetic field strength should have a distribution of a decreasing function of the distance from the Earth. Hence, the total magnetic field strength reverses before the substorm onset. Soon after onset, at 02:24 UT, the total magnetic field strengths at both the THA and THE locations had larger changes and significant fluctuation.

The plasma pressures determined by ESA plasma pressure tensor data are shown in Figure 4. Because both the ion and electron pressure tensors are symmetric matrices at each moment, with a $3 \mathrm{~s}$ time resolution, we diagonalized each matrix for both the ion

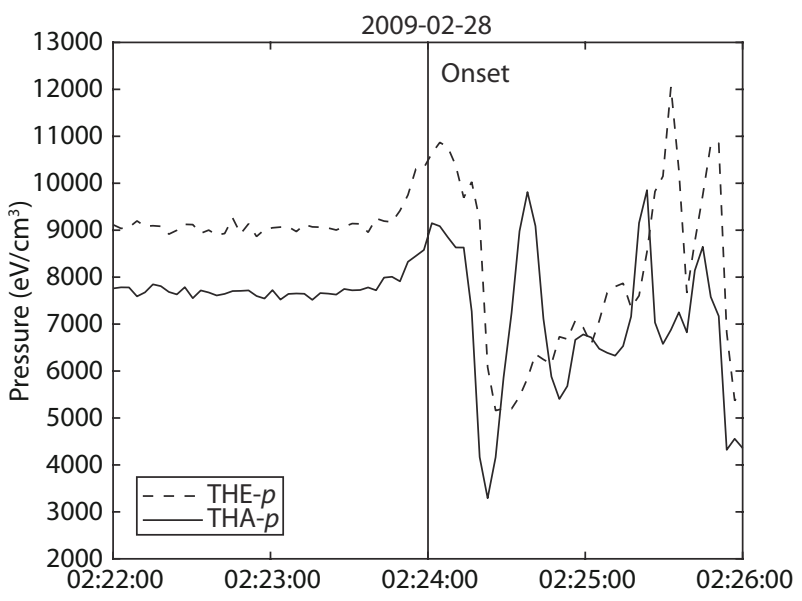

Figure 4. Plasma pressures from THEMIS THA and THE around 02:24 UT on February 28, 2009. The time range is 2 minutes before and after the substorm onset, the same format as in Figure 3 . The solid line is the THA plasma pressure, and the dashed line is the THE plasma pressure. The solid black vertical line indicates the substorm onset time at 02:24 UT. The onset time is determined by the $A E$ index, with a resolution of one minute. 
and electron pressure tensor at each moment. We then added the diagonal elements together as the ion and electron pressure value, and finally summarized the ion and electron pressure value as the total pressure. The plasma pressure was about 7,544 eV/cm at the THA location and about $9,012 \mathrm{eV} / \mathrm{cm}^{3}$ at the THE location before substorm onset. In addition, the pressure underwent a violent change after substorm onset.

From Equation (3), we know that entropy is proportional to pressure and inversely proportional to $5 / 3$ power of the magnetic field. In this event of the substorm onset at $~ 02: 24$ UT on February 28,2009 , the flux tube at the THA location had a larger magnetic field and a smaller plasma pressure than those of the flux tube at the THE location. Assuming the lengths of the flux tubes passing through THA and THE were almost the same (as can be seen from the bottom of Figure 3, the spacing of the two neighboring flux tubes passing through the two THEMIS satellites is 0.1 $R_{\mathrm{E}}$, whereas the two satellites are located at $8.7 R_{\mathrm{E}}$ ), we calculated the entropy at the locations of the two satellites by using Equation (3). As shown in Figure 5, entropy at the THA location was smaller than that at the THE location before substorm onset. Hence, the entropy at the outer bubble of the THA location was lower than the entropy at the inner bubble of the THE location before the substorm onset. According to our theory, the outer bubble has lower entropy than that of the inner bubble, forming an unstable domain. The development of interchange or ballooning instability manifests as a turbulent evolution and significant fluctuation (Duan SP et al., 2021) of the magnetic field, plasma pressure, and entropy shortly after the onset at 02:24 UT, as shown in Figures 3-5.

Figure 6 shows the ion density using ESA ion density data, with a 3 $s$ time resolution. The ion density is in order of magnitude consistent with the $0.4 / \mathrm{cm}^{3}$ given by Xing $X$ et al. (2012). lon bulk velocities are shown in Figures 7 and 8 for THA and THE. Here, a righthanded orthogonal system and a magnetic meridian plane

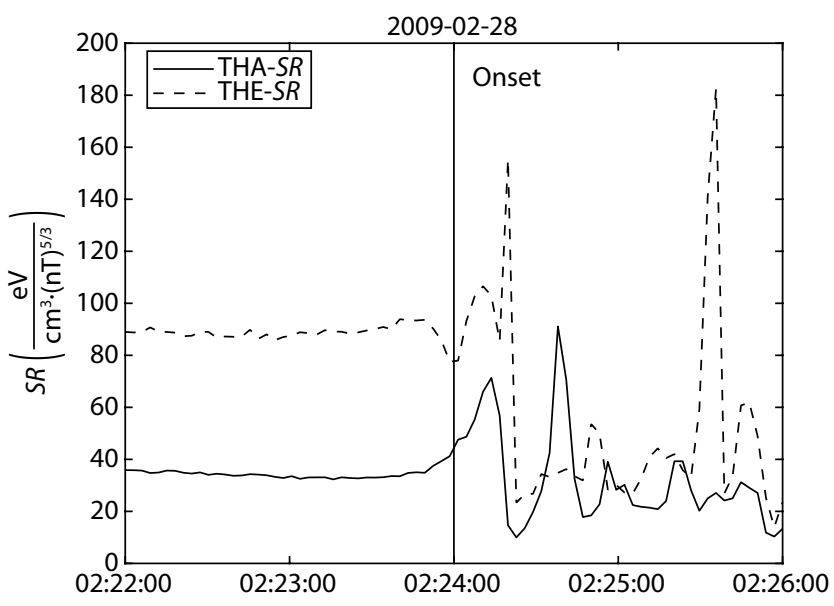

Figure 5. Entropy (related) at the THA and THE locations around 02:24 UT on February 28, 2009, the same format as in Figure 2. The solid line is the THA entropy (related), and the dashed line is the THE entropy (related). The solid black vertical line indicates the substorm onset time at $\sim 02: 24$ UT. The onset time is determined by the $A E$ index, with a resolution of one minute.

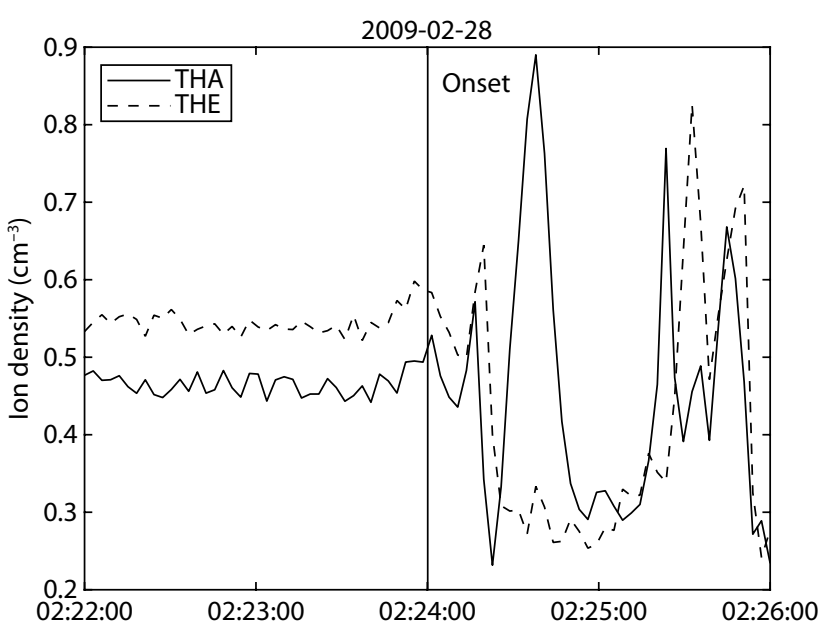

Figure 6. The ion density from THEMIS THA and THE around 02:24 UT on February 28, 2009. The time range is 2 minutes before and after the substorm onset, the same format as in Figure 3 . The solid line is the THA ion density, and the dashed line is the THE ion density. The solid black vertical line indicates the substorm onset time at $\sim 02: 24$ UT. The onset time is determined by the $A E$ index, with a resolution of one minute.

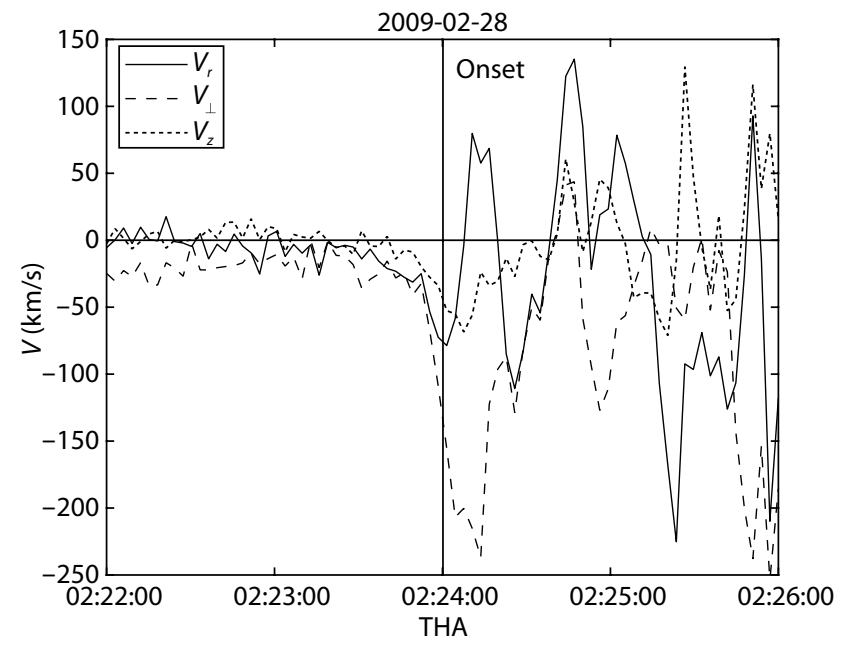

Figure 7. The ion bulk velocity from THEMIS THA around 02:24 UT on February 28,2009 . The time range is 2 minutes before and after the substorm onset, the same format as in Figure 3. The solid line indicates the $V_{r}$ component, the dashed line represents the $V_{\perp}$ component, and the dotted line represents the $V_{z}$ component. The vertical solid black line indicates the substorm onset time at $\sim 02: 24$ UT. The onset time is determined by the $A E$ index, with a resolution of one minute.

passing through the satellite are used. The direction pointing from the Earth to the satellite is defined as the $V_{r}$ component direction. The $V_{\perp}$ component direction is perpendicular to this magnetic meridian plane and is pointed in a west to east direction. The $V_{z}$ component direction is determined from the $V_{r}$ direction and the $V_{\perp}$ direction by the right-handed orthogonal system. Before onset, flow at the THA location is very weak. Shortly after onset, the $V_{r}$ component shows a moderate earthward flow of 


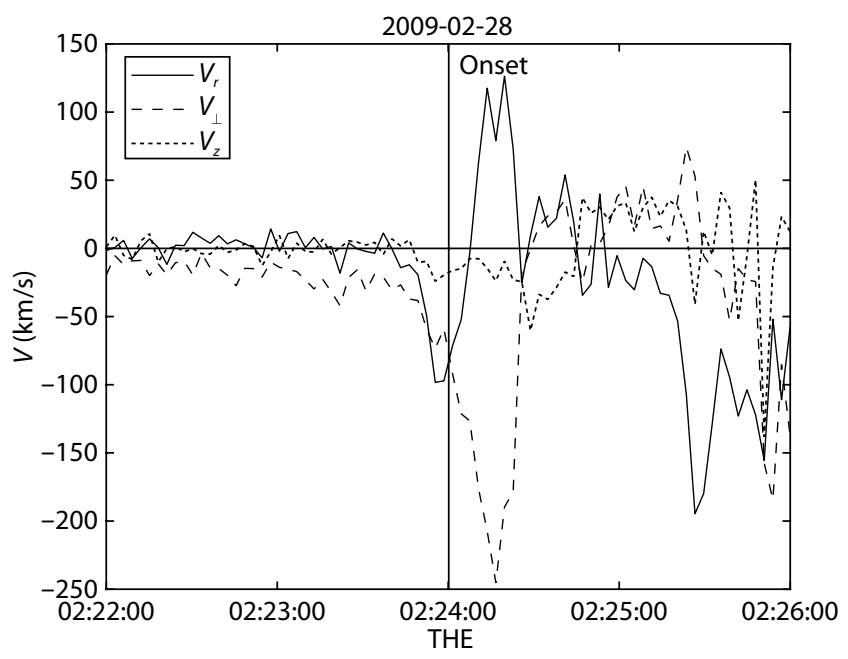

Figure 8. The ion bulk velocity from THEMIS THE around 02:24 UT on February 28, 2009. The other settings are the same as in Figure 7. $\sim 50 \mathrm{~km} / \mathrm{s}$, followed by a turbulent fluctuation in the next 2 minutes. At the THE location, a similar flow behavior was observed. The only exception is the time of first appearance of the earthward flow at this location of the magnetosphere, which is slightly ahead of that of the aurora.

Figure 9 shows the all-sky-camera aurora images from the ground station Kuujjuaq (KUUJ) according to the satellite footprints on the ionosphere shown in Figure $2 \mathrm{a}$, which has a time range from 02:18 to 02:29 UT on February 28, 2009, during the period of the substorm. We can see that there is an arc before the substorm onset, which brightens suddenly. No streamer could be found before onset.

\subsection{The Event on February 22, 2008, with a Preceding Streamer}

In this subsection, we selected the event at 09:29 UT on February 22, 2008, observed by THEMIS THD and THE (Nishimura et al., 2011, supporting information Data Set S1). THEMIS THD and THE

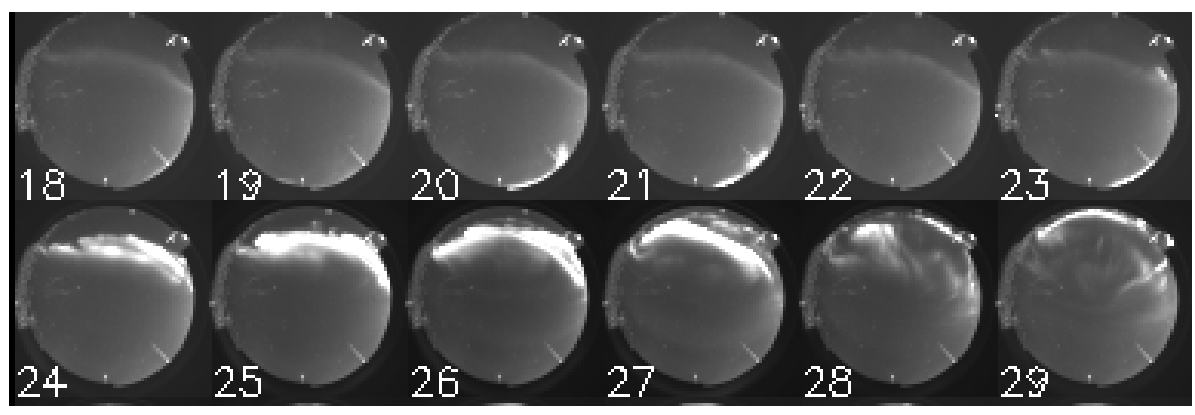

Figure 9. All-sky-camera aurora observations from the KUUJ station used to demonstrate that the onset seen at THEMIS THA and THE coincided with expansion of the substorm onset. The time range is from 02:18 to 02:29 UT on February 28, 2009.

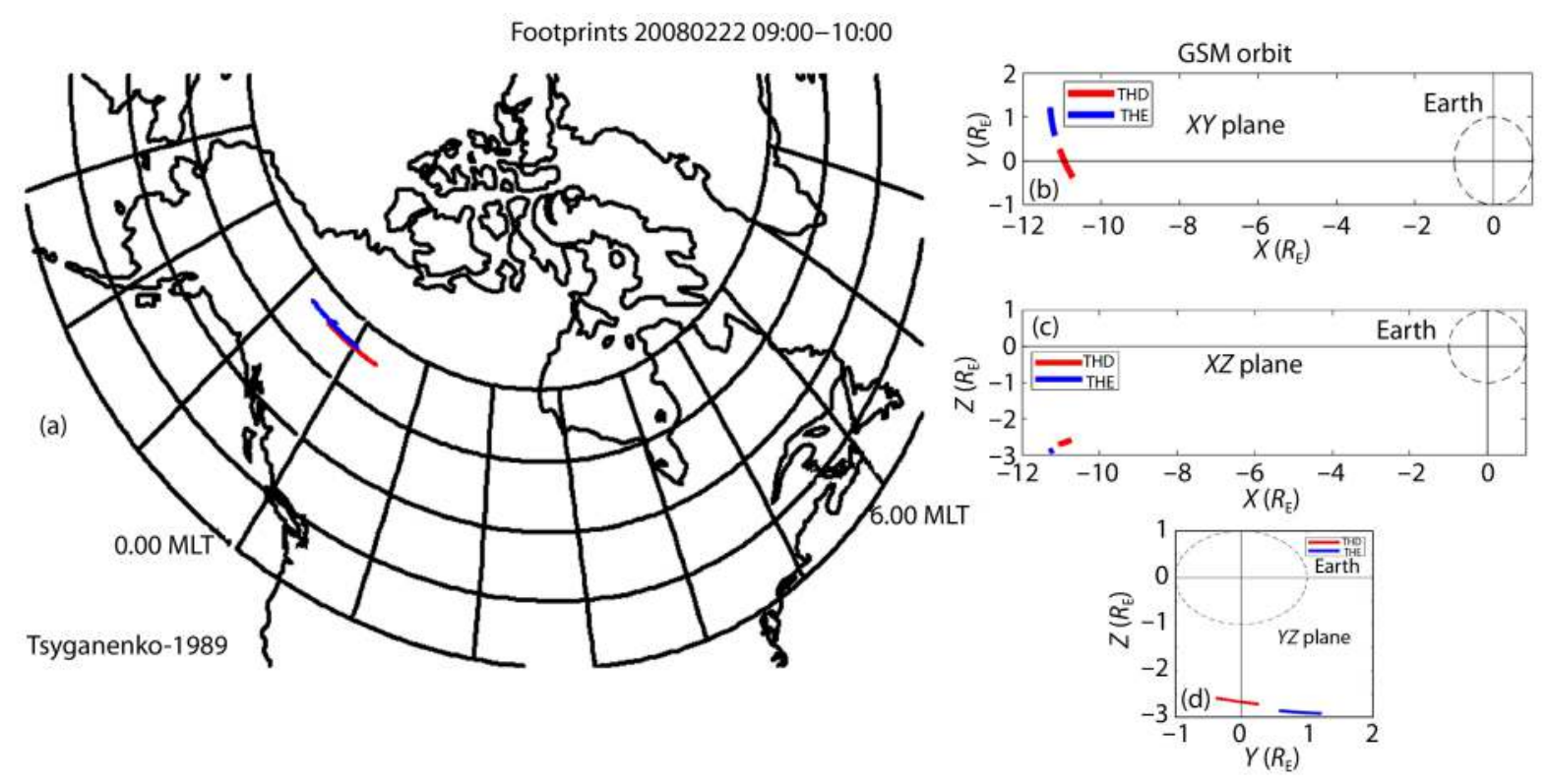

Figure 10. THEMIS THD (red) and THE (blue) satellite footprints as projected on the Earth's ionosphere (a) for February 22, 2008, 09:00 to 10:00 UT, and their orbits in GSM coordinates $(b-d)$. The footprints are individually projected along the local magnetic field line as described by the model of Tsyganenko (1989). The $0.00 \mathrm{MLT}$ in (a) indicates midnight. The orbits of the THD and THE satellites in the $X Y, X Z, Y Z$ plane of the GSM coordinate system are shown in (b), (c), and (d). The red line indicates the THD orbit, and the blue line is the THE orbit. 
were at $(X, Y, Z)_{\mathrm{GSM}}=(-10.91,-0.05,-2.66) R_{\mathrm{E}}$ and $(-11.26,0.90$, -2.89) $R_{\mathrm{E}}$ at 09:29 UT. Their footprints as projected on the Earth's ionosphere and orbits in the $X Y, X Z, Y Z$ plane of the GSM coordinate system are shown in Figure 10. Satellite THE was located slightly farther away from the Earth than THD, as shown in Figures 10b, 10c, 10d, and 11 (labels under the $x$-axis). Satellite THE was located more poleward than THD when projected from the equatorial plane to the ionosphere, as shown in Figure 10a. Both satellites were located near midnight. In addition, the two spacecraft were separated by $4.73^{\circ}$ in azimuth; thus, the two satellites were roughly in line with the Earth.

Figure 11 shows the total magnetic field strengths for this event from THD and THE. The data resolution and the figure format are the same as in Figure 3. The total magnetic field strength of THD was smaller than that of THE before the substorm onset, meaning there was a total magnetic field strength reversal. The particle

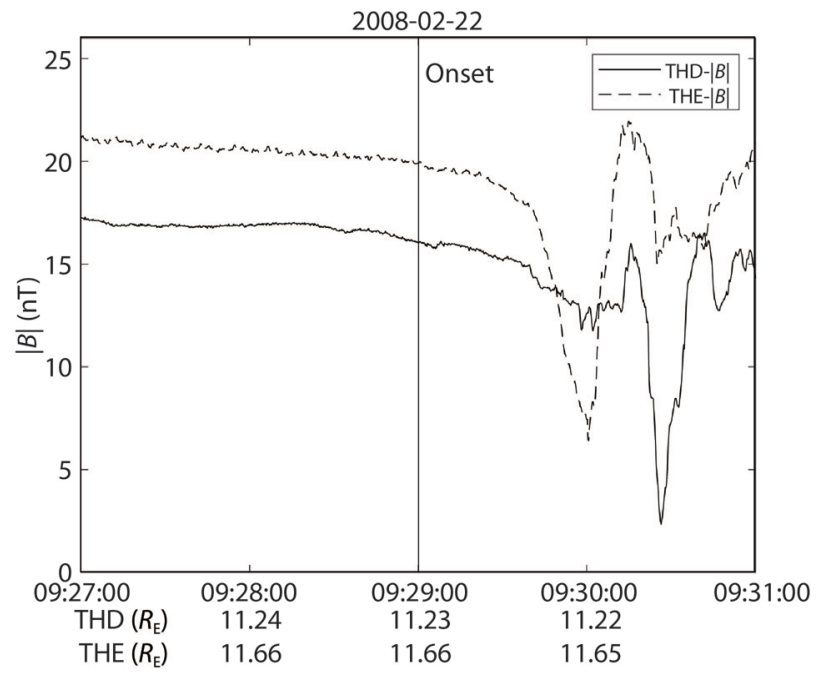

Figure 11. The total magnetic field strengths from THEMIS THD and THE in GSM coordinates around 09:29 UT on February 22, 2008. The other settings are the same as in Figure 3.

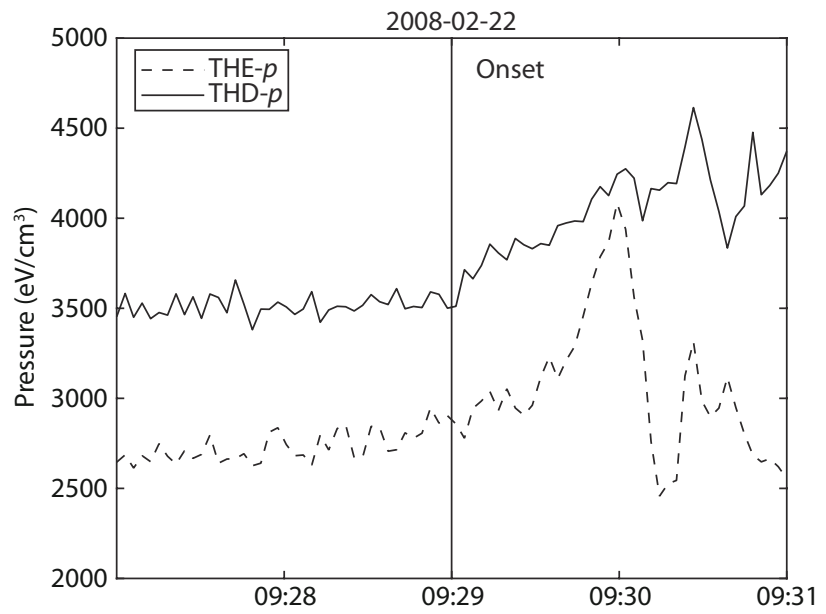

Figure 12. The plasma pressures from THEMIS THD and THE around 09:29 UT on February 22, 2008. The other settings are the same as in Figure 4.

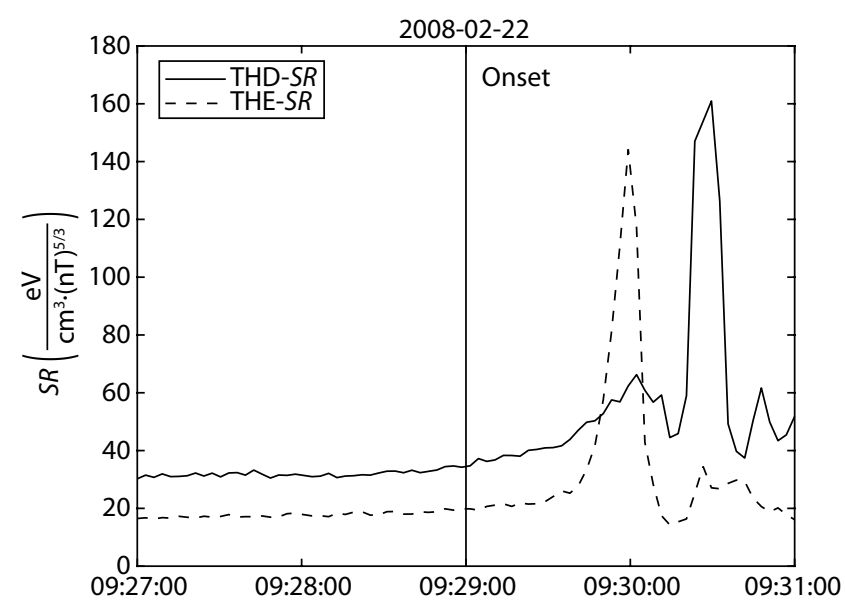

Figure 13. Entropy (related) at the THD and THE locations around 09:29 UT on February 22, 2008. The other settings are the same as in Figure 5.

pressure at the THD location was larger than that at the THE location, as shown in Figure 12. The entropy values at THD and THE are shown in Figure 13, with the assumption that the lengths of the flux tubes passing through THD and THE were almost the same. (This can be seen at the bottom of Figure 11, where the spacing of the two neighboring flux tubes passing through the two THEMIS satellites is $0.4 R_{\mathrm{E}}$, whereas the two satellites are located at $11.4 R_{\mathrm{E}}$.) Thus, we can obtain similar information as with the first event that the entropy was less at the THE location than at the THD location. Figures 11 and 13 could also convey the message that an unstable domain existed just before substorm onset because of the total magnetic field strength reversal and entropy reversal. The ion density using ESA ion density data is given in Figure 14. The plasma flows at the two satellite regions are shown in Figures 15 and 16. After the substorm onset, all quantities fluctuated.

The all-sky-camera images from the Inuvik (INUV) station, which were chosen according to the satellite footprints from Figure 10a,

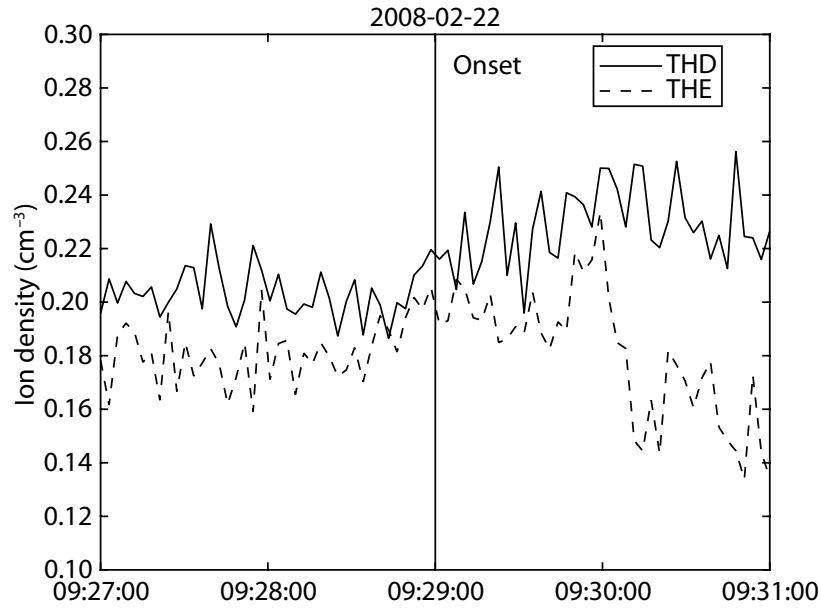

Figure 14. The ion density from THEMIS THD and THE around 09:29 UT on February 22, 2008. The other settings are the same as in Figure 6. 


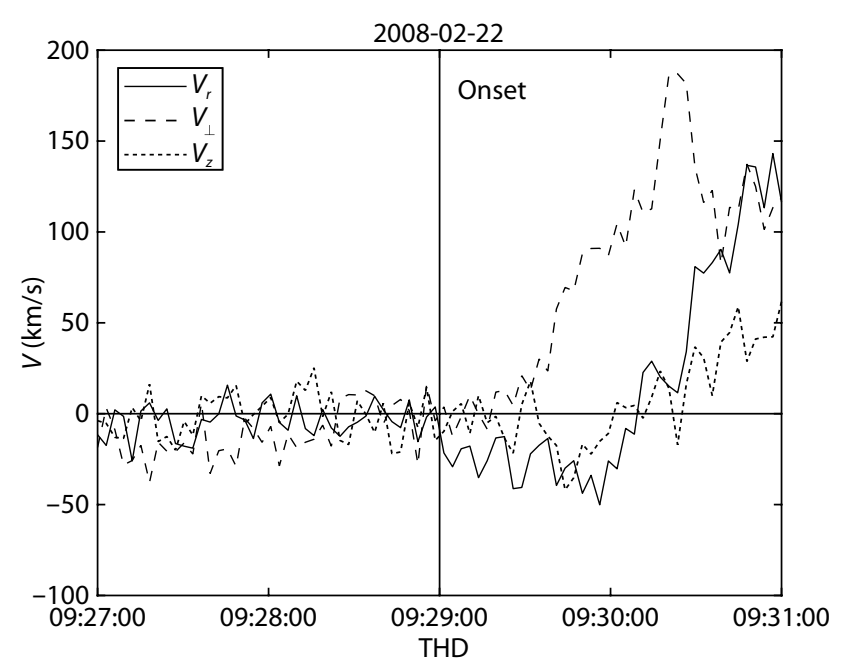

Figure 15. The ion bulk velocity from THEMIS THD around 09:29 UT on February 22, 2008. The other settings are the same as in Figure 7. We used the same coordinate system as for the first event.

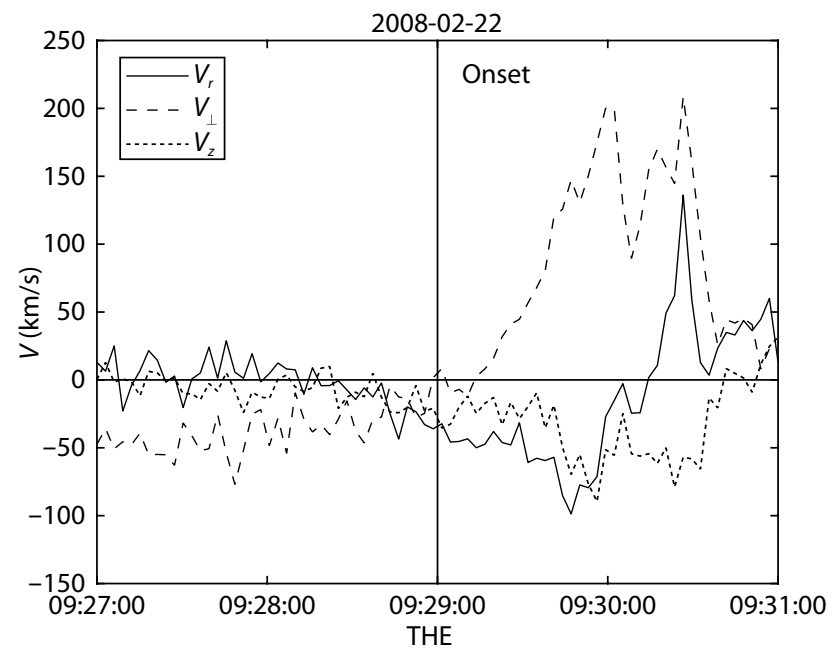

Figure 16. The ion bulk velocity from THEMIS THE around 09:29 UT on February 22, 2008. The other settings are the same as in Figure 7. are shown in Figure 17 with a time range from 09:24 to 09:35 UT on February 22, 2008. A thin arc appears before the substorm onset. A streamer moves equatorward toward the arc, leading to the substorm onset (from the all-sky image in Figure 17). As can be seen, the streamer is initially in the north-south direction accompanying a flow burst in the magnetosphere, then its direction turns to east-west as its accompanying flow changes the earthward movement to a spreading in the azimuthal direction.

\section{Statistical Analysis}

To ascertain whether the total magnetic field strength and entropy reversal are common phenomena for substorm onset, we performed a statistical study. We adopted the time range of 2 minutes before and after substorm onset, as for the previous two event case studies. Table S1 in the supporting information provides the 205 substorm onset events selected.

For all 205 events, we found an occurrence rate of the total magnetic field strength reversal of $69 \%$ (141 events) and an occurrence rate of entropy reversal of $77 \%$ (158 events). The results are shown in Figure 18.

An important task before us is how to interpret the occurrence rates of the total magnetic field strength reversal (69\%) and entropy reversal (77\%). Several factors may have affected the resulting values. First, the criteria used for the event selection may be crucial. The events were chosen when the THEMIS satellites were in a location between $\sim 8$ and $\sim 12 R_{\mathrm{E}}$ downtail distance, and the magnetic local time of the satellite location also needed to be at 3 hours before and after midnight. We then searched for a possible magnetic reversal among the three satellites THA, THE, and THD. Second, the separation among THEMIS satellites may have played a role here. The spacing between satellites could be as narrow as $0.1 R_{\mathrm{E}}$ or as wide as $1.5 R_{\mathrm{E}}$. Third, the tailward dimension of an unstable domain, which was less than $1 R_{\mathrm{E}}$, may have had a strong interactive effect with the separation of the satellites. Two conclusions can be drawn from the above considerations. One is that the total magnetic field strength reversal was dominant before the substorm onset. Another is that the total magnetic field strength reversal occurred mostly in the region between $\sim 8$ and $\sim 12 R_{\mathrm{E}}$, especially in the region between $\sim 11$ and $\sim 12 R_{\mathrm{E}}$.

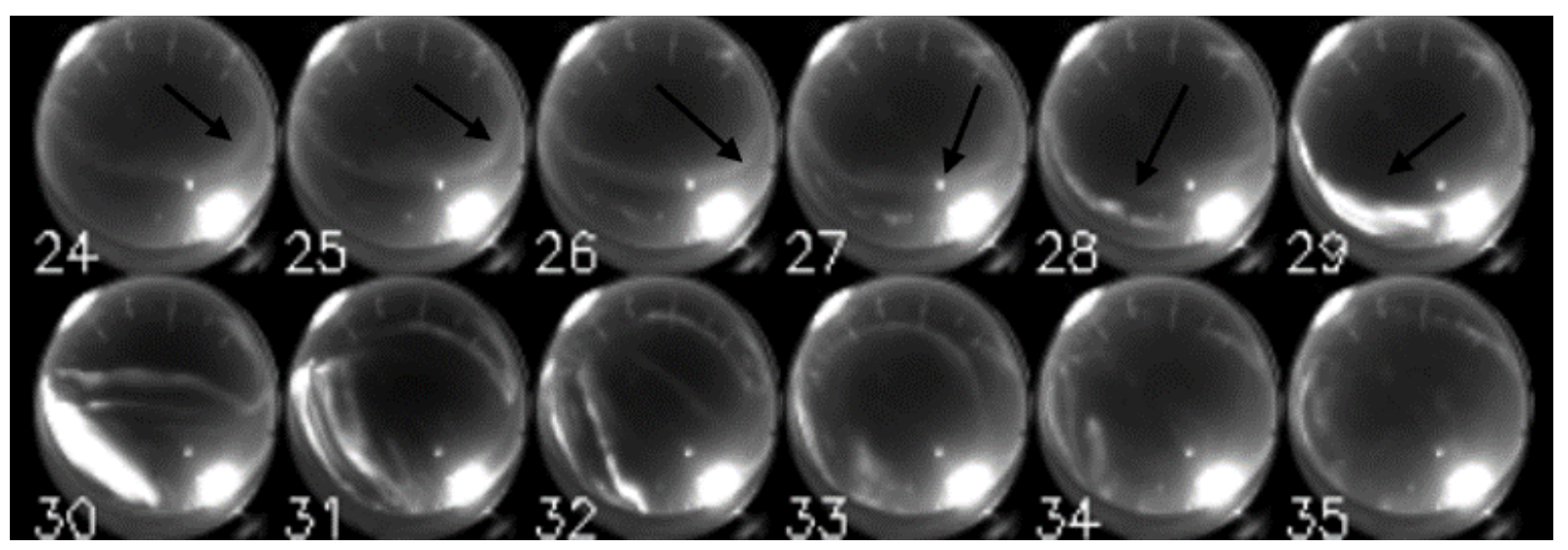

Figure 17. Aurora images from the all-sky-camera at the INUV station. The black arrow indicates a streamer. The time range is from 09:24 to 09:35 UT on February 22, 2008. 


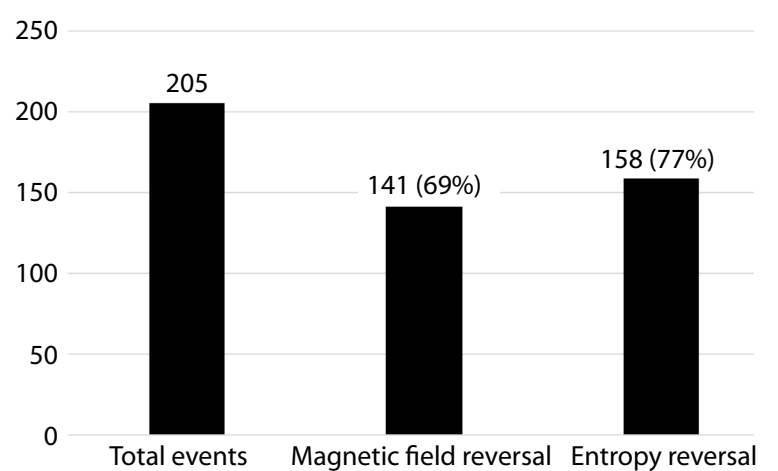

Figure 18. Statistical results for the total 205 events. The occurrence rate of the total magnetic field strength reversal was $69 \%$ (141 events), and the occurrence rate of entropy reversal was $77 \%$ (158 events)

The difference between the occurrence rates of the total magnetic field strength reversal (69\%) and entropy reversal (77\%) can be explained by the procedure used in calculating entropy. The approximation of almost the same lengths of the magnetic flux tubes passing through the two satellites under consideration may have yielded a lower entropy value of the tailward satellite than reality when the separation of the two satellites was relative larger (e.g., $>1 R_{\mathrm{E}}$ ). There were also some events in which the difference in the total magnetic field strength was very small without reversal but the plasma pressure had a decreasing distribution tailward, causing entropy reversal.

We also aimed to identify whether a streamer appeared before substorm onset. However, we were not able to obtain an accurate result because the all-sky-camera imagers were not clear enough to identify a streamer.

\section{Discussion and Conclusions}

In this study, to verify the entropy switch model of substorm onset proposed by Chen CX (2016), we conducted two case studies by examining two events in detail, one with and one without a streamer before substorm onset. We then performed a statistical study using 205 events.

The two events selected revealed many features of substorm onset. Before substorm onset, the total magnetic field strength on the tailward side of the domain should be larger than that on the earthward side of the domain, whereas the distribution of entropy should be reversed that of the total magnetic field strength. Such a domain is interchange or ballooning unstable. The development of the interchange or ballooning instability will cause the substorm onset. Magnetic field, plasma pressure, entropy, and velocity will fluctuate following the onset.

Because an interchange or ballooning unstable domain may be formed by a larger scale magnetospheric convection (without a streamer) or isolated flows (with a streamer), we used all-sky-camera imagers to check the appearance of a streamer. Because of the lack of clarity and resolution of the data, we could not distinguish well whether a streamer occurred before substorm onset for most cases.
For a more complete perspective, we would like to make comparisons with other similar studies, such as those by Xing $X Y$ et al. (2013) and Zhu P et al. (2009). These authors focused on ballooning instability, which has long been proposed as the instability that initiates substorm onset.

Interchange instability and ballooning instability are similar in some respects and different in others. Ballooning instability in a general sense is also a kind of interchange instability. Observationally, interchange instability and ballooning instability share the same aurora wave structures investigated by Xing $X Y$ et al. (2013). From the aurora phenomena, these two instabilities are indistinguishable. In Figures 4 and 12, an earthward pressure gradient appears before substorm onset. This pressure gradient was also observed by Xing XY et al. (2013) and numerically simulated by Zhu P et al. (2009).

Although interchange instability and ballooning instability share some similarities, they are two different instabilities. Interchange instability involves an entropy reversal, and entropy is a global quantity that is characterized by a whole magnetic flux tube. Conversely, ballooning instability requires a locally strong pressure gradient and "bad" curvature of the magnetic field, which favors a stretched plasma sheet.

Currently, it is quite a challenging task to distinguish by observations exactly which instability is responsible for substorm onset. The entropy switch model of substorm onset (which pays attention to interchange instability) has merit, as it unifies the opposite models of outside-in and inside-out. From a more macroscopic view, if sequences of transport of increasing entropy and decreasing entropy exist, substorm onset must be the turning point from one sequence to another.

In summary, we found observational evidence for the entropy switch model of substorm onset. Two detailed events were investigated, one with a preceding streamer and the other without, which clearly revealed the total magnetic field strength and entropy reversal before a substorm onset. A statistical analysis using 205 events yielded an occurrence rate of the total magnetic field strength reversal (69\%) and an occurrence rate of entropy reversal (77\%).

\section{Acknowledgments}

This work was supported by the National Natural Science Foundation of China (Grant No. NSFC41974204). The THEMIS data set can be accessed online through http://themis.ssl.berkeley.edu/.

\section{References}

Angelopoulos, V. (2008). The THEMIS mission. Space Sci. Rev., 141(1-4), 5-34. https://doi.org/10.1007/s11214-008-9336-1

Auster, H. U., Glassmeier, K. H., Magnes, W., Aydogar, O., Baumjohann, W., Constantinescu, D., Fischer, D., Fornacon, K. H., Georgescu, E., ... Wiedemann, M. (2008). The THEMIS fluxgate magnetometer. Space Sci. Rev., 141(1-4), 235-264. https://doi.org/10.1007/s11214-008-9365-9

Chen, C. X., and Wolf, R. A. (1993). Interpretation of high-speed flows in the plasma sheet. J. Geophys. Res.: Space Phys., 98(A12), 21409-21419. https://doi.org/10.1029/93ja02080

Chen, C. X., and Wolf, R. A. (1999). Theory of thin-filament motion in Earth's magnetotail and its application to bursty bulk flows. J. Geophys. Res.: Space 
Phys., 104(A7), 14613-14626. https://doi.org/10.1029/1999JA900005

Chen, C. X. (2013). Theoretical constraints on the cross-tail width of bursty bulk flows. Ann. Geophys., 31(12), 2179-2192. https://doi.org/10.5194/angeo-312179-2013

Chen, C. X. (2016). Substorm onset: a switch on the sequence of transport from decreasing entropy to increasing entropy. Geophys. Res. Lett., 43(10), 4834-4840. https://doi.org/10.1002/2016GL069114

Chen, C. X., and Wang, C. P. (2019). Contribution of patchy reconnection to the ion-to-electron temperature ratio in the Earth's magnetotail. Earth Planet. Phys., 3(6), 474-480. https://doi.org/10.26464/epp2019049

Chen, C. X. (2021). Preservation and variation of ion-to-electron temperature ratio in the plasma sheet in geo-magnetotail. Earth Planet. Phys., 5(4), 337-347. https://doi.org/10.26464/epp2021035

Duan, S. P., Wang, C., Liu, W. W., and He, Z. H. (2021). Characteristics of magnetic dipolarizations in the vicinity of the substorm onset region observed by THEMIS. Earth Planet. Phys., 5(3), 239-250. https://doi.org/10.26464/epp2021031

Henderson, M. G., Reeves, G. D., and Murphree, J. S. (1998). Are north-south aligned auroral structures an ionospheric manifestation of bursty bulk flows?. Geophys. Res. Lett., 25(19), 3737-3740. https://doi.org/10.1029/98GL02692

Hones, E. W. Jr. (1977). Substorm processes in the magnetotail: comments on 'On hot tenuous plasmas, fireballs, and boundary layers in the Earth's magnetotail' by L. A. Frank, K. L. Ackerson, and R. P. Lepping. J. Geophys. Res., 82(35), 5633-5640. https://doi.org/10.1029/JA082i035p05633

Lui, A. T. Y. (1991). A synthesis of magnetospheric substorm models. J. Geophys. Res.: Space Phys., 96(A2), 1849-1856. https://doi.org/10.1029/90JA02430

Lui, A. T. Y. (2011). Reduction of the cross-tail current during near-Earth dipolarization with multisatellite observations. J. Geophys. Res.: Space Phys., 116(A12), A12239. https://doi.org/10.1029/2011JA017107

Lyons, L. R., Wang, C. P., Nagai, T., Mukai, T., Saito, Y., and Samson, J. C. (2003). Substorm inner plasma sheet particle reduction. J. Geophys. Res.: Space Phys., 108(A12), 1426. https://doi.org/10.1029/2003JA010177

McFadden, J. P., Carlson, C. W., Larson, D. Ludlam, M., Abiad, R., Elliott, B., Turin, P., Marckwordt, M., and Angelopoulos, V. (2008). The THEMIS ESA plasma instrument and in-flight calibration. Space Sci. Rev., 141(1-4), 277-302. https://doi.org/10.1007/s11214-008-9440-2

Mende, S. B., Harris, S. E., Frey, H. U., Angelopoulos, V., Russell, C. T., Donovan, E., Jackel, B., Greffen, M., and Peticolas, L. M. (2008). The THEMIS array of ground-based observatories for the study of auroral substorms. Space Sci. Rev., 141(1-4), 357-387. https://doi.org/10.1007/s11214-008-9380-x
Nishimura, Y., Lyons, L., Zou, S., Angelopoulos, V., and Mende, S. (2010). Substorm triggering by new plasma intrusion: THEMIS all-sky imager observations. J. Geophys. Res.: Space Phys., 115(A7), A07222. https://doi.org/10.1029/2009JA015166

Nishimura, Y., Lyons, L. R., Angelopoulos, V., Kikuchi, T., Zou, S., and Mende, S. B. (2011). Relations between multiple auroral streamers, pre-onset thin arc formation, and substorm auroral onset. J. Geophys. Res.: Space Phys., 116(A9), A09214. https://doi.org/10.1029/2011JA016768

Pontius, D. H. Jr., and Wolf, R. A. (1990). Transient flux tubes in the terrestrial magnetosphere. Geophys. Res. Lett., 17(1), 49-52. https://doi.org/10.1029/GL017i001p00049

Sergeev, V. A., Liou, K., Meng, C. I., Newell, P. T., Brittnacher, M., Parks, G., and Reeves, G. D. (1999). Development of auroral streamers in association with localized impulsive injections to the inner magnetotail. Geophys. Res. Lett., 26(3), 417-420. https://doi.org/10.1029/1998GL900311

Toffoletto, F. R., Spiro, R. W., Wolf, R. A., Birn, J., and Hesse, M. (2000). Computer experiments on substorm growth and expansion. In Proceedings of the 5th International Conference on Substorms (pp. 351-356). St. Petersburg, Russia: ESA.

Tsyganenko, N. A. (1989). A magnetospheric magnetic field model with a warped tail current sheet. Planet. Space Sci., 37(1), 5-20. https://doi.org/10.1016/0032-0633(89)90066-4

Wolf, R. A., Toffoletto, F. R., Spiro, R. W., Hesse, M., and Birn, J. (2002). Magnetospheric substorms: an inner-magnetospheric modeling perspective. COSPAR Colloq. Ser., 12, 221-229. https://doi.org/10.1016/S0964-2749(02)80221-9

Wolf, R. A., Wan, Y. F., Xing, X., Zhang, J. C., and Sazykin, S. (2009). Entropy and plasma sheet transport. J. Geophys. Res., 114(A9), A00D05. https://doi.org/10.1029/2009JA014044

Xing, X., Lyons, L. R., Zhou, X. Z., Angelopoulos, V., Donovan, E., Larson, D., Carlson, C., and Auster, U. (2012). On the formation of pre-onset azimuthal pressure gradient in the near-Earth plasma sheet. J. Geophys. Res., 117(A8), A08224. https://doi.org/10.1029/2012JA017840

Xing, X. Y., Liang, J., Spanswick, E., Lyons, L., and Angelopoulos, V. (2013). Auroral wave structures and ballooning instabilities in the plasma sheet. $J$. Geophys. Res.: Space Phys., 118(10), 6319-6326. https://doi.org/10.1002/2013JA019068

Zhu, P., Raeder, J., Germaschewski, K., and Hegna, C. C. (2009). Initiation of ballooning instability in the near-Earth plasma sheet prior to the 23 March 2007 THEMIS substorm expansion onset. Ann. Geophys., 27(3), 1129-1138. https://doi.org/10.5194/angeo-27-1129-2009 


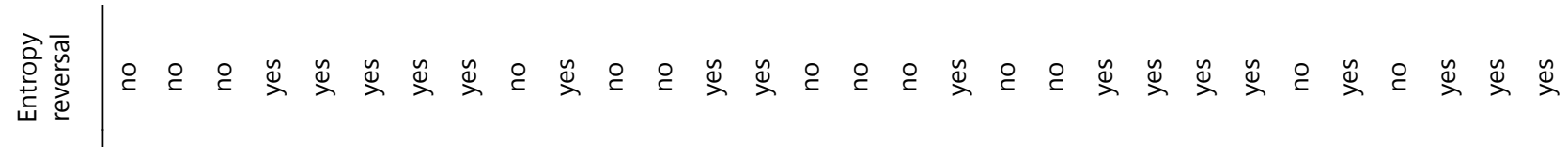

केष

点

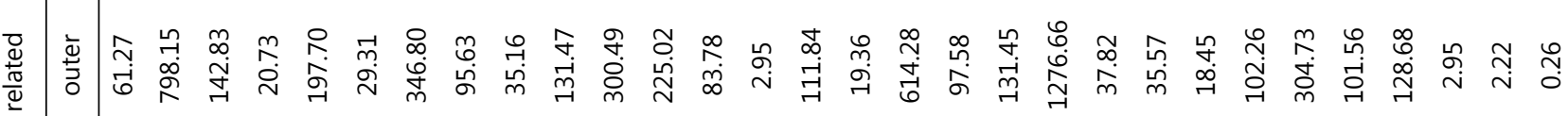

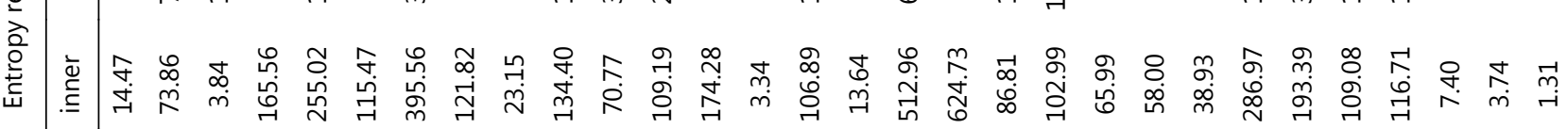

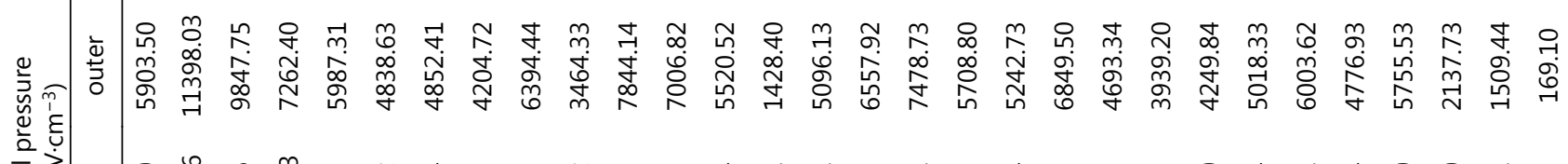
焉

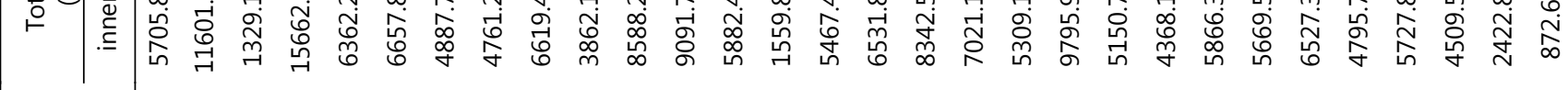

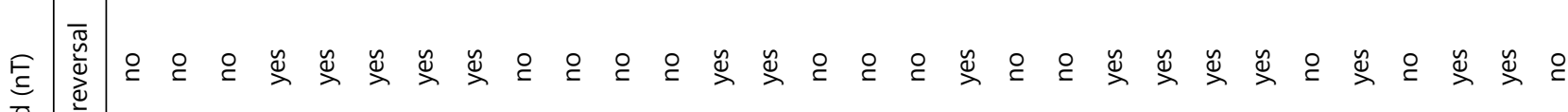
毫 矛 वे

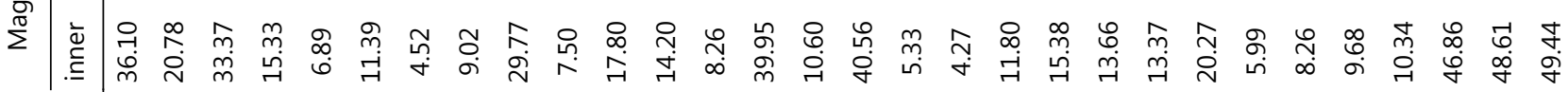

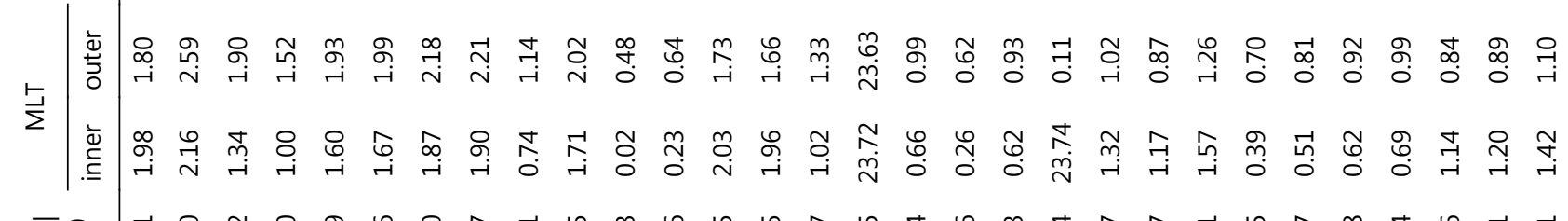

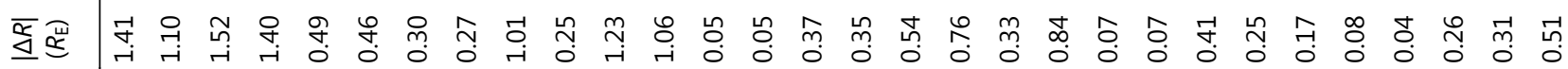

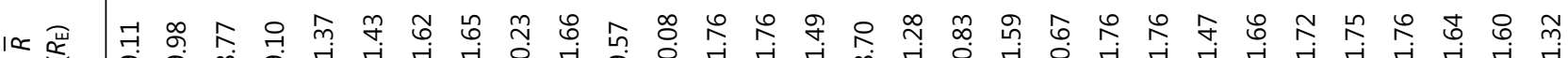

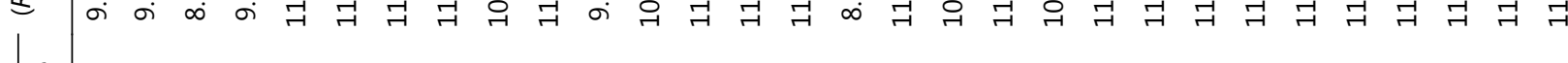

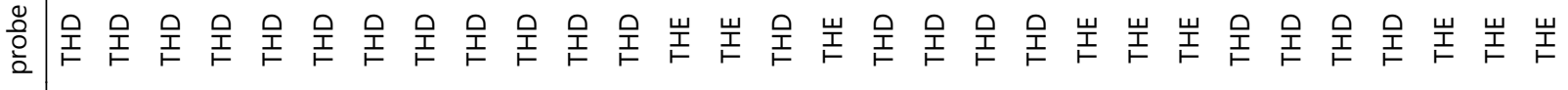
巴) 产 ๖)

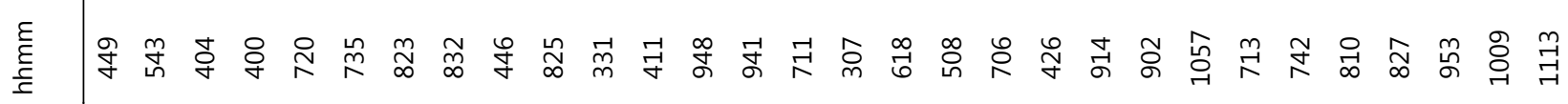

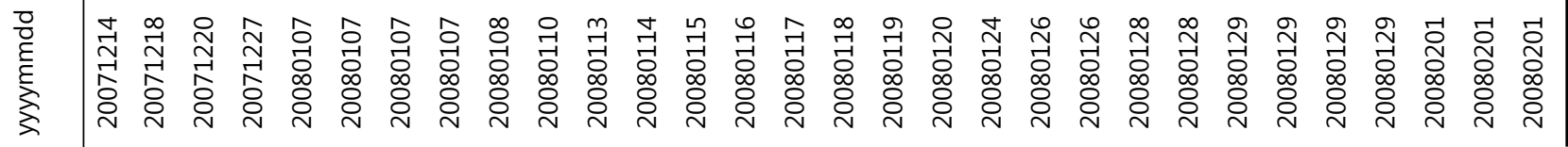


京焉端

高

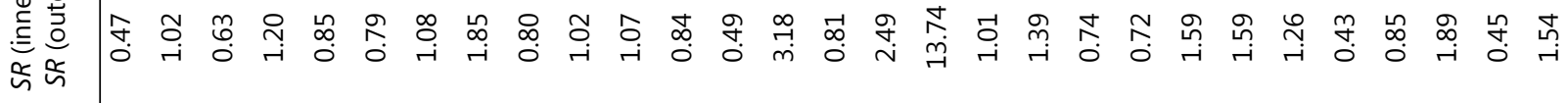

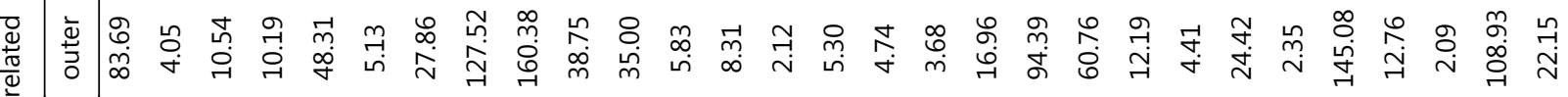

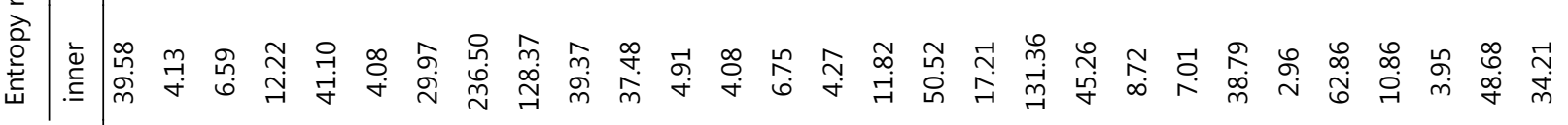

-

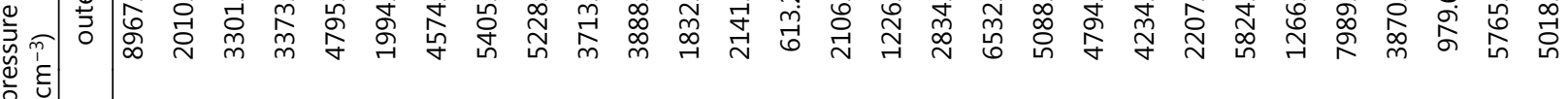

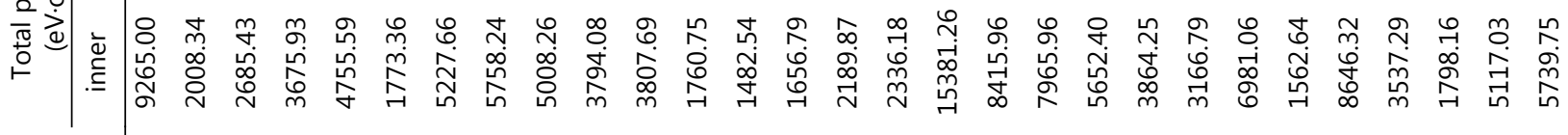

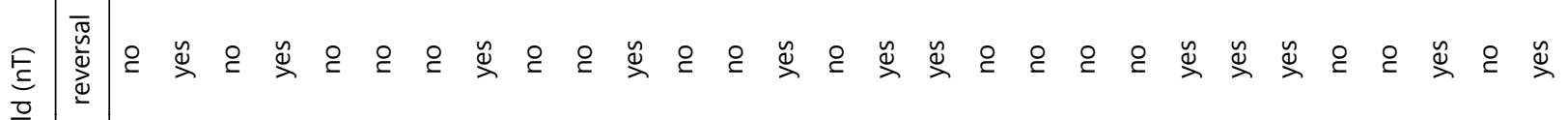

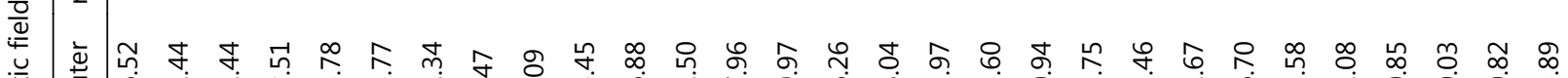

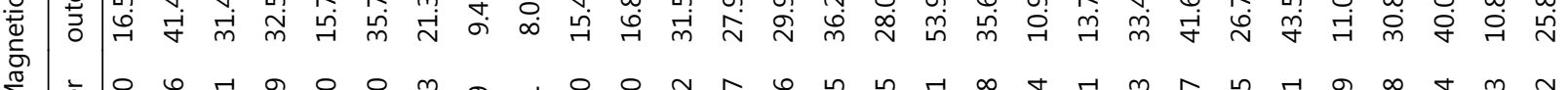

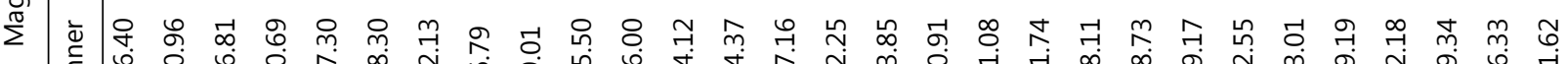
至

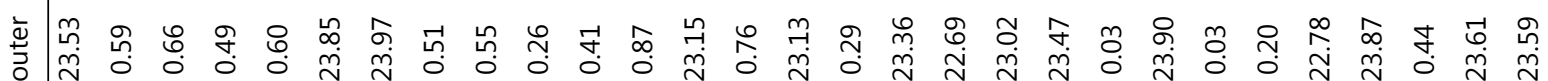

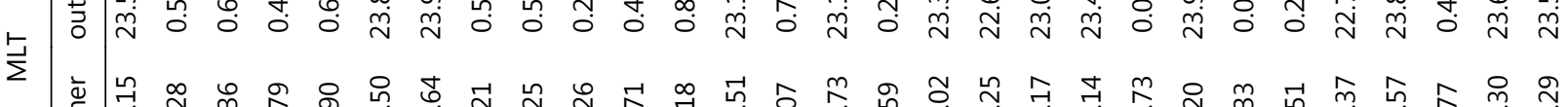

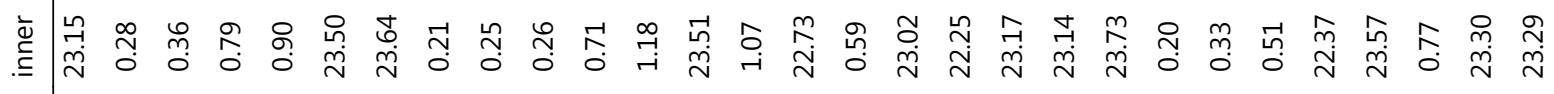

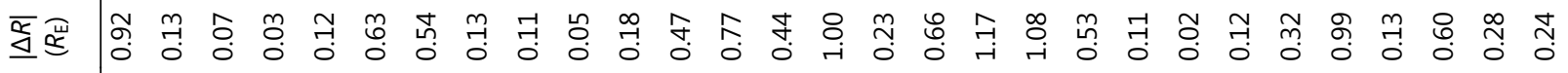

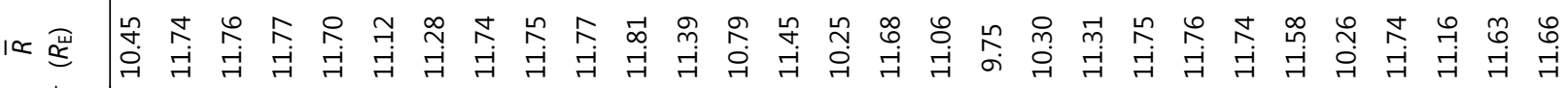

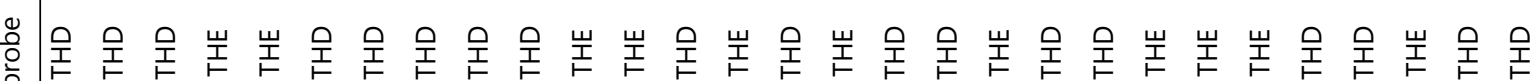
Q ळँ

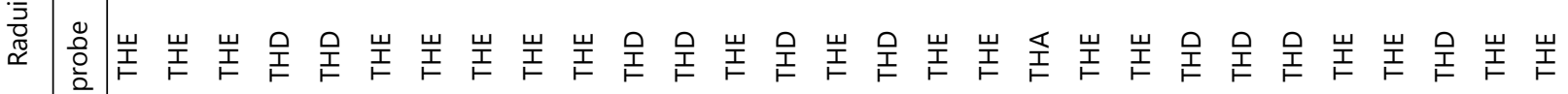

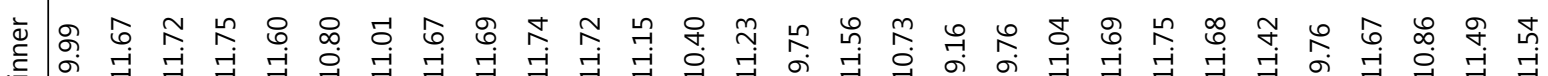

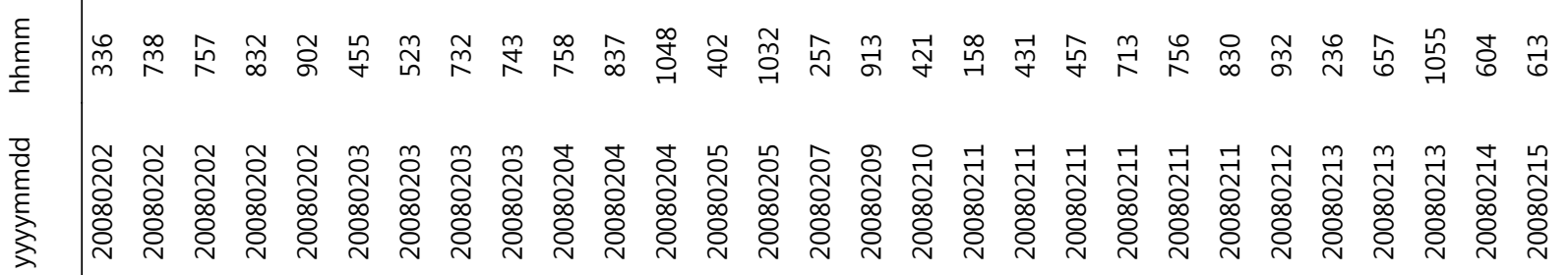




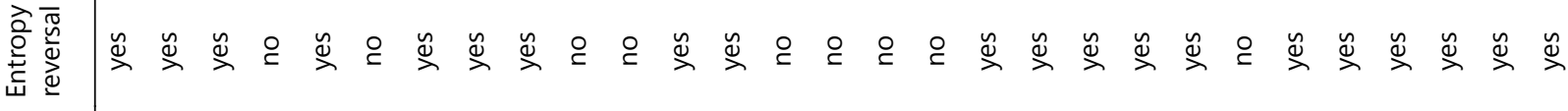

产

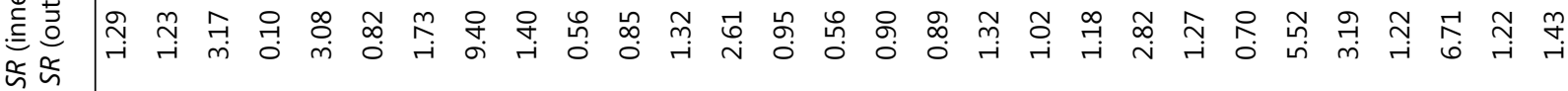

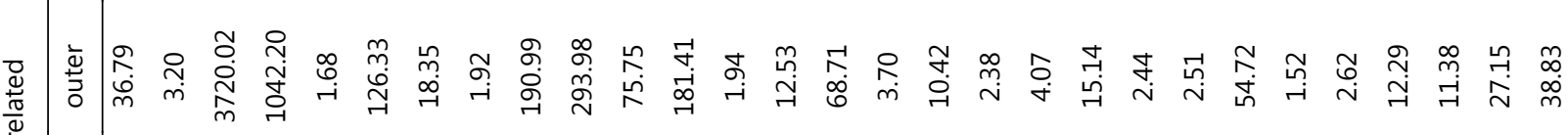

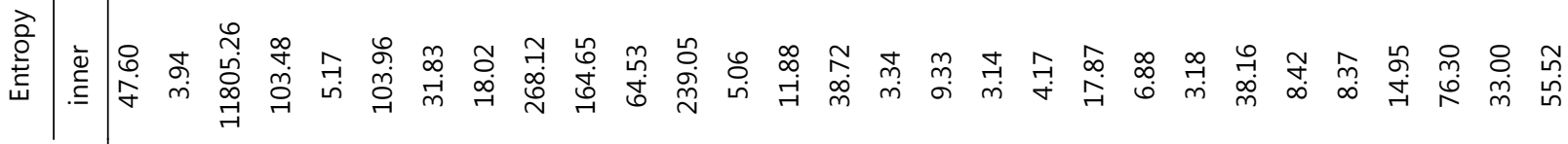

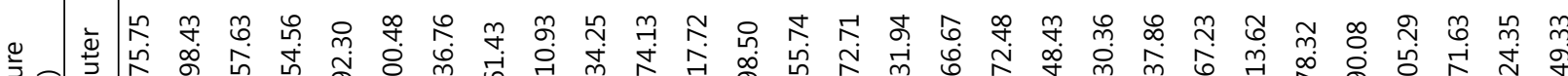

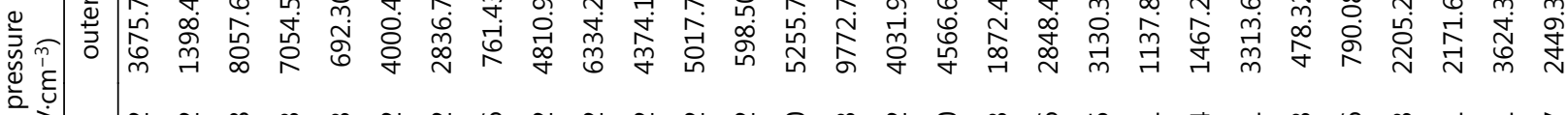

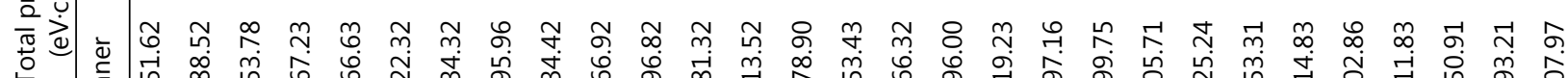

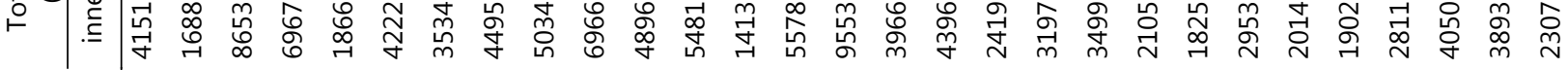

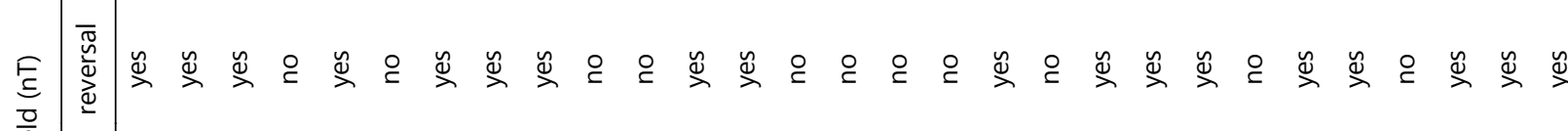

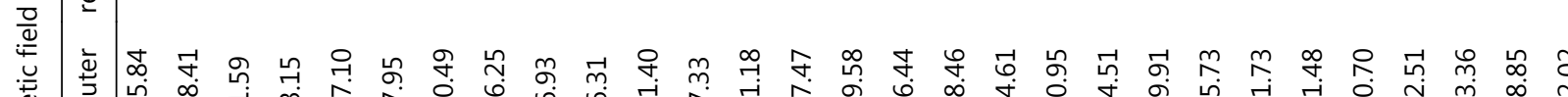

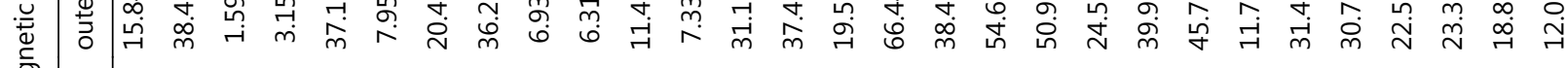

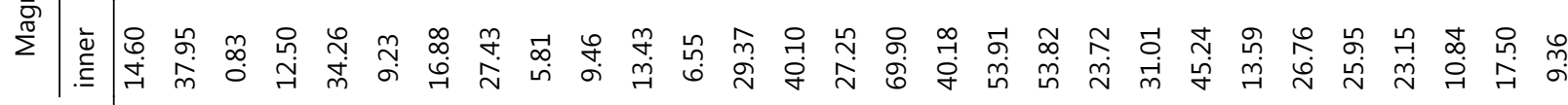

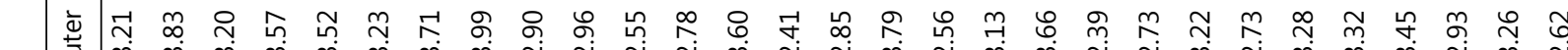

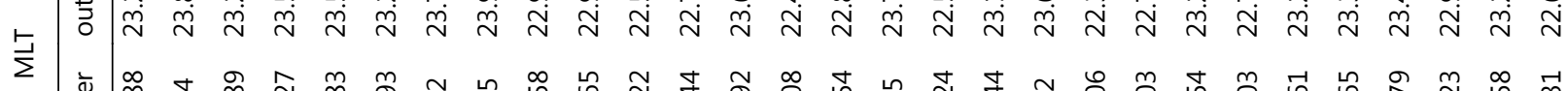

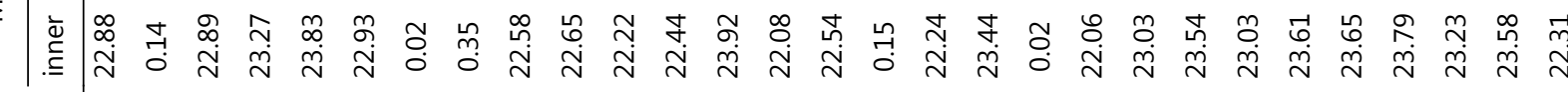
迋巡

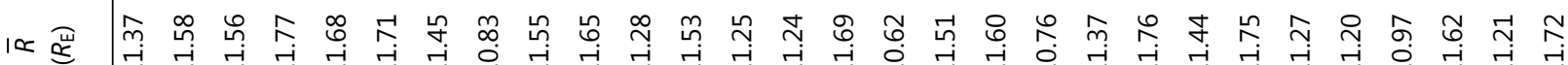

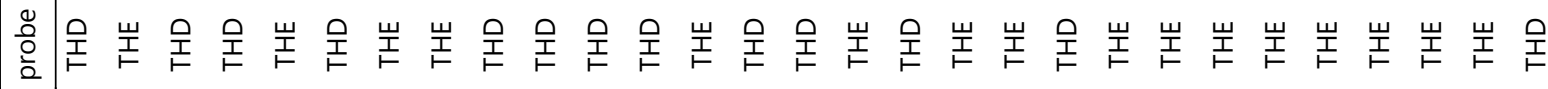

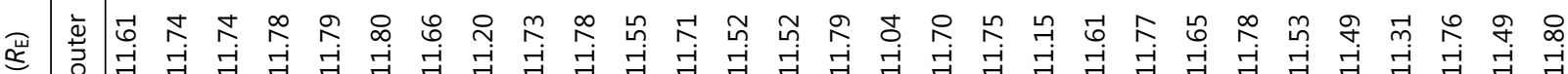

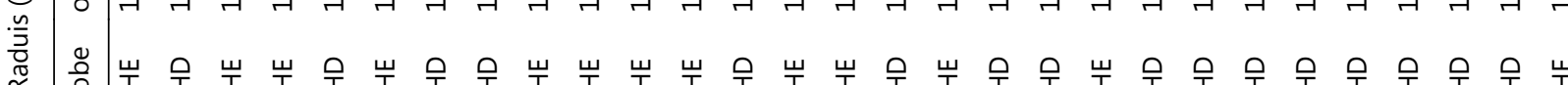
造

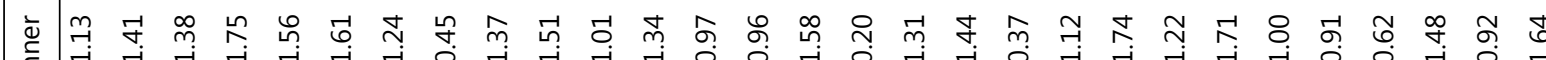

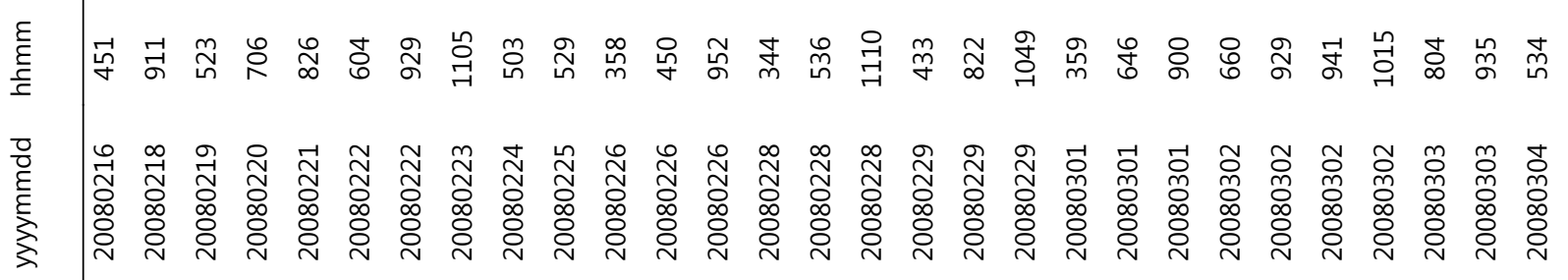




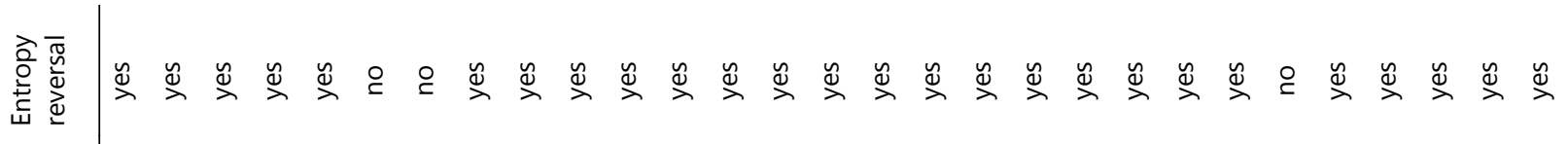

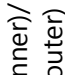

象里

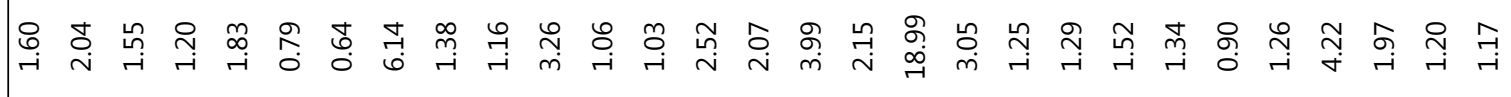

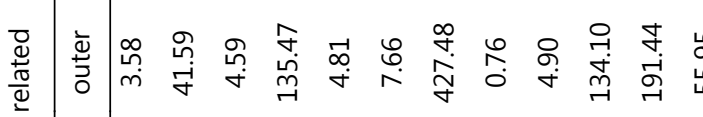

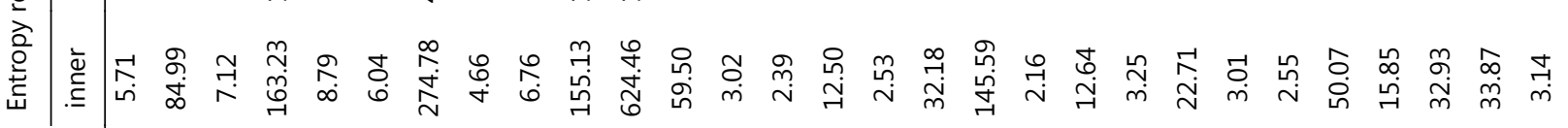

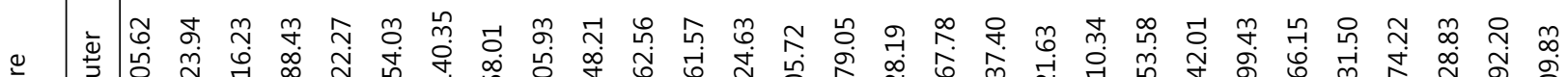

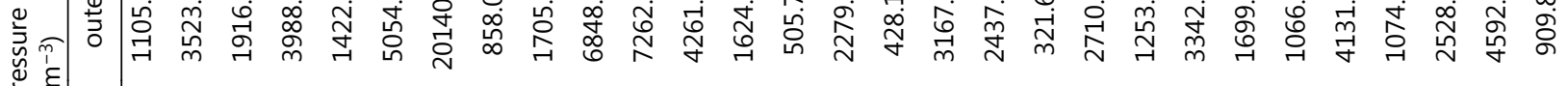
远 E

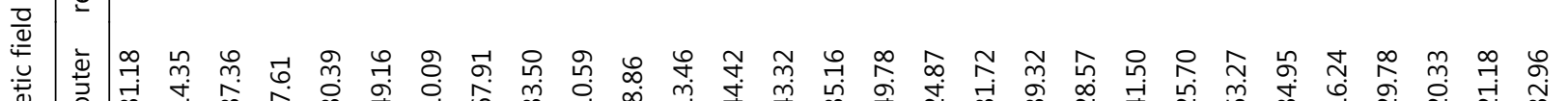
苞

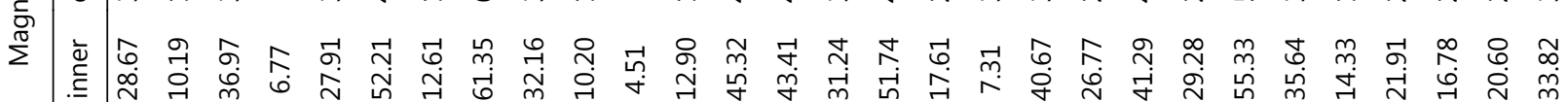

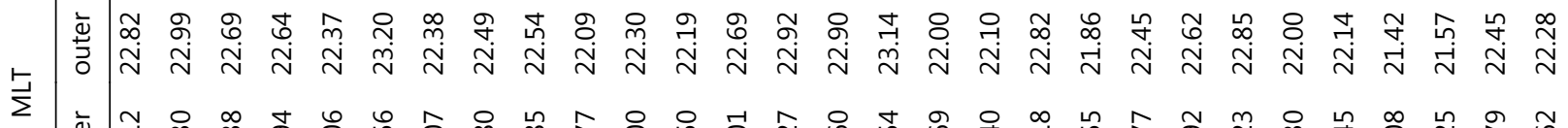

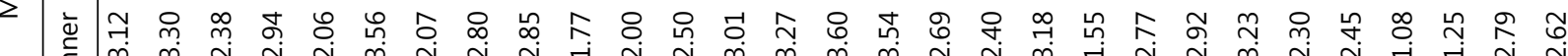

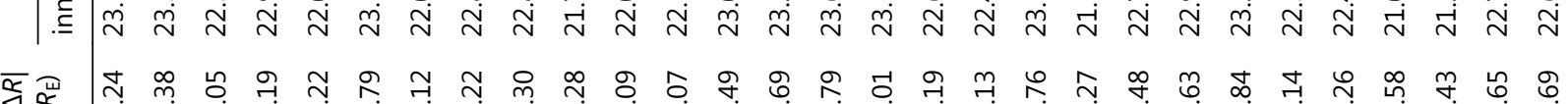

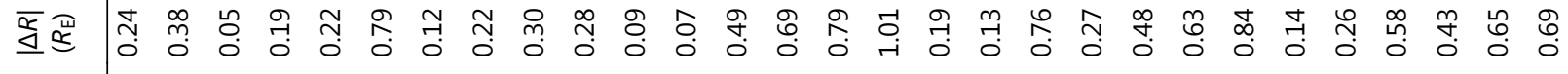

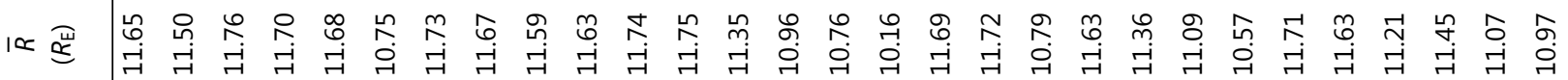

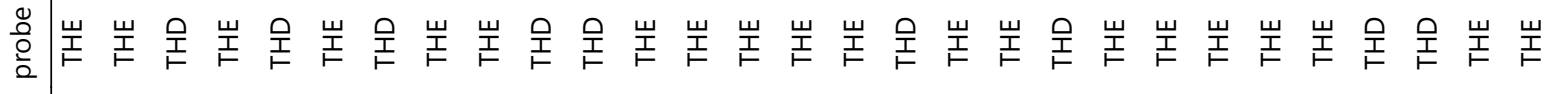

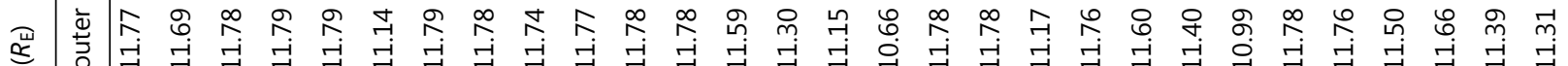
号 离 ๘

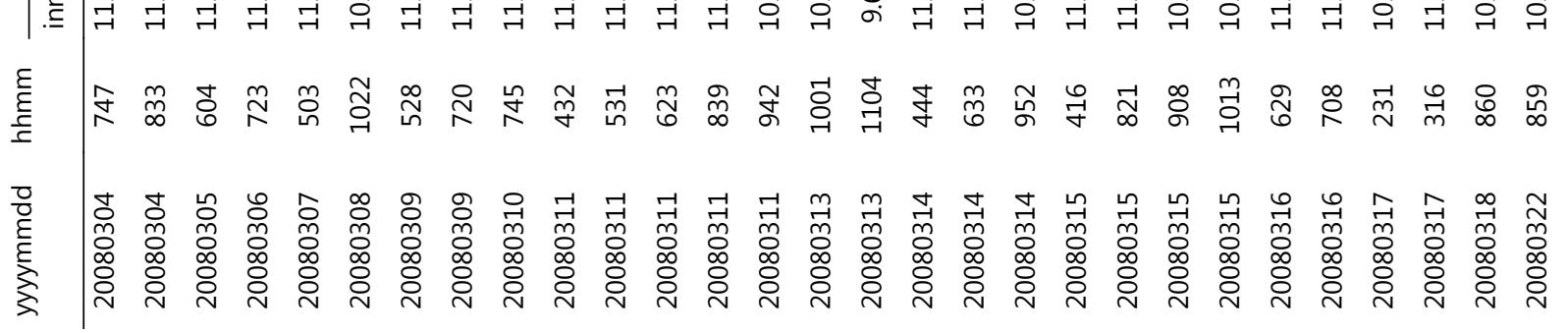




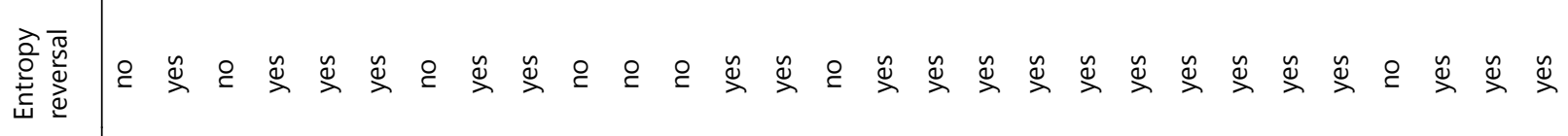

彥

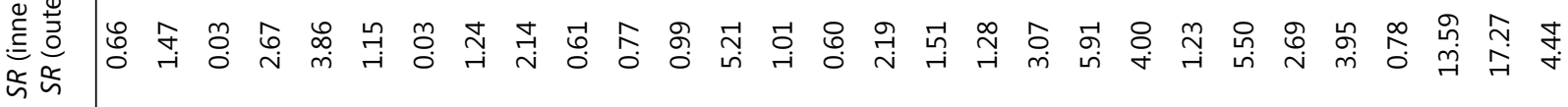

离

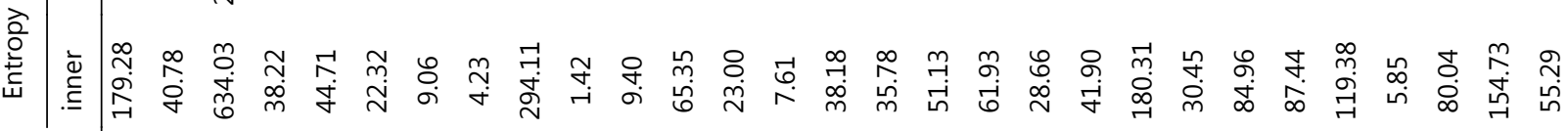

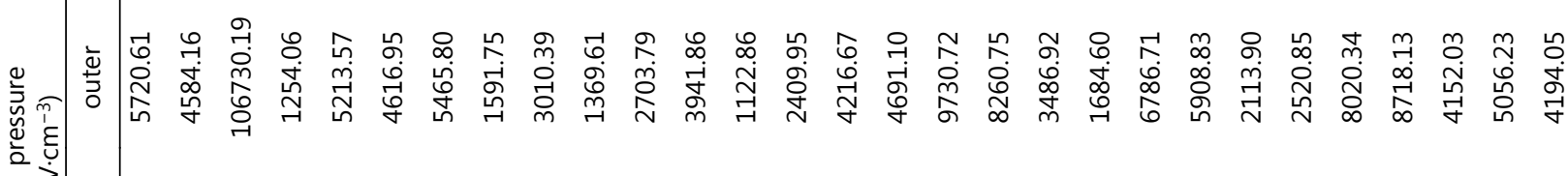

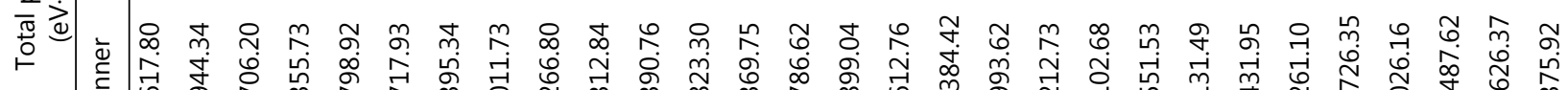

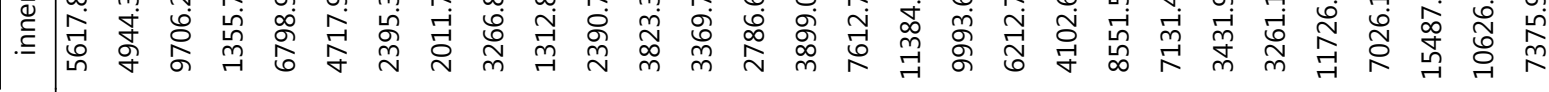

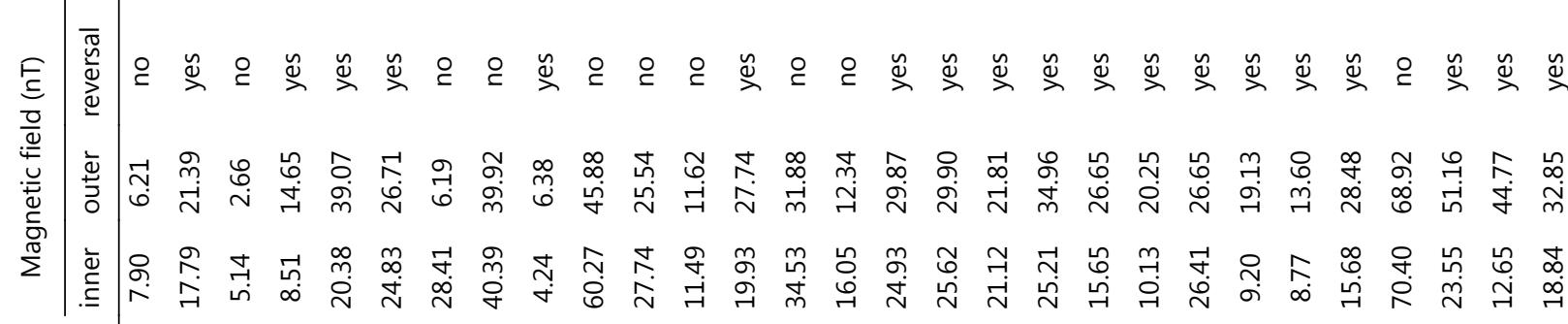

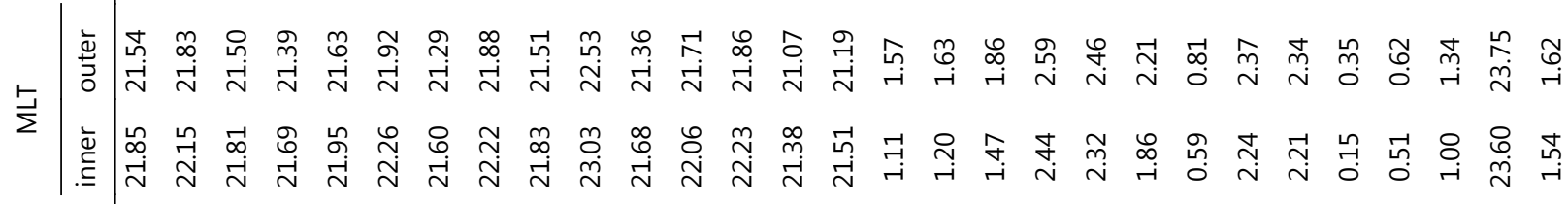

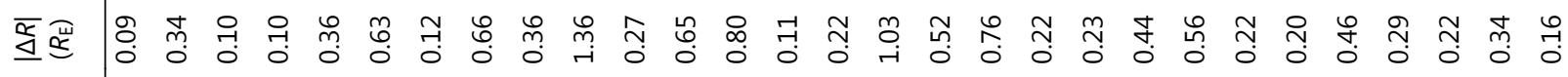

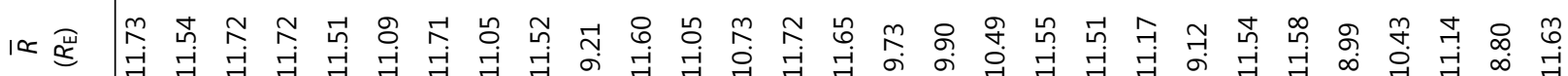

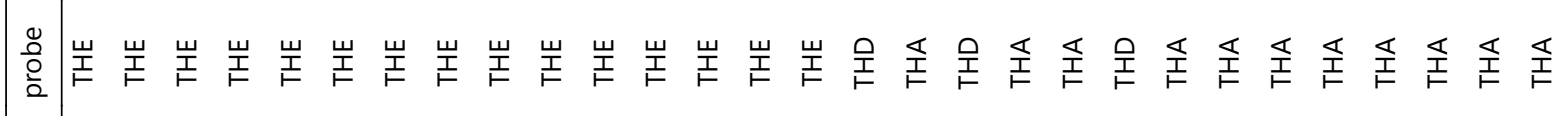

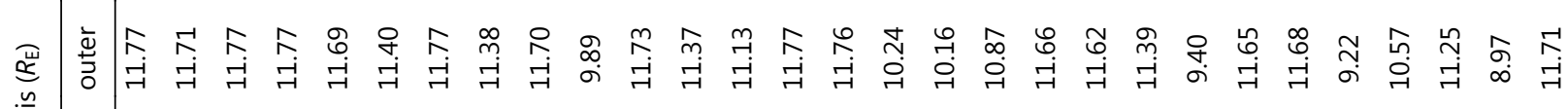
言

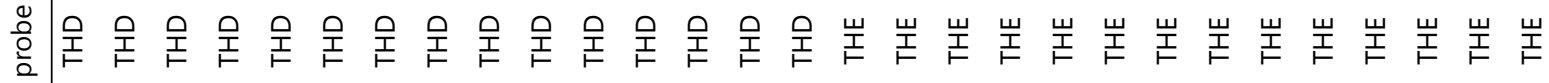

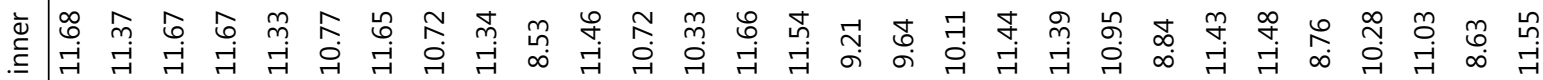

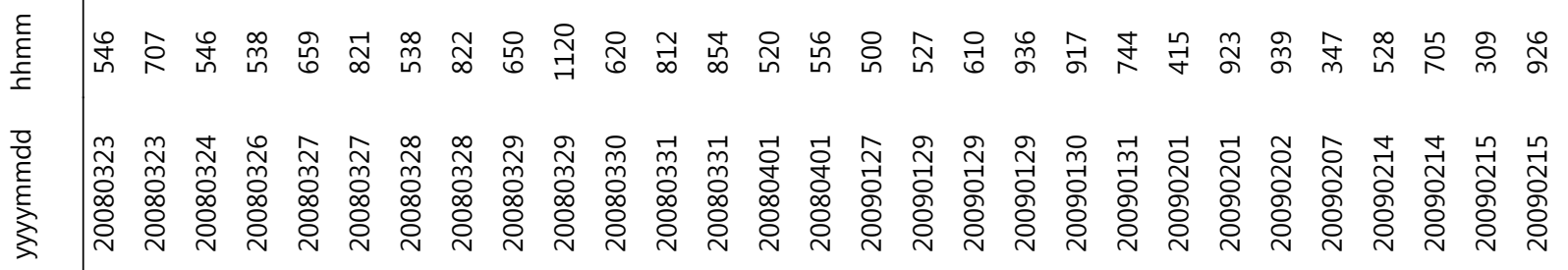




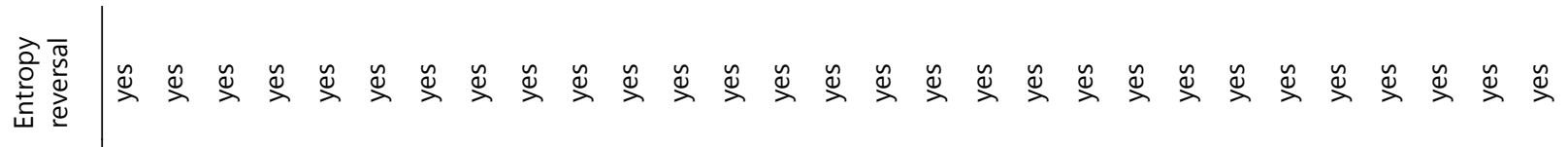

言言

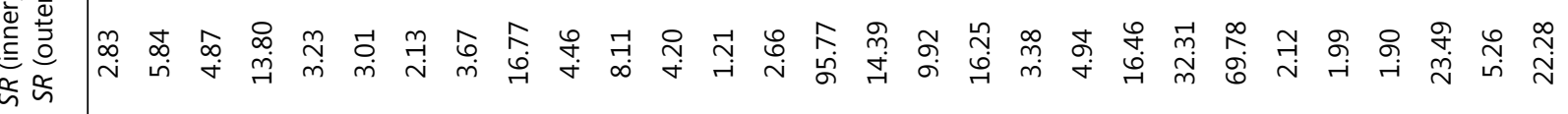

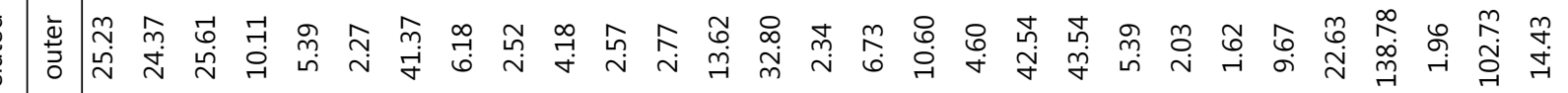

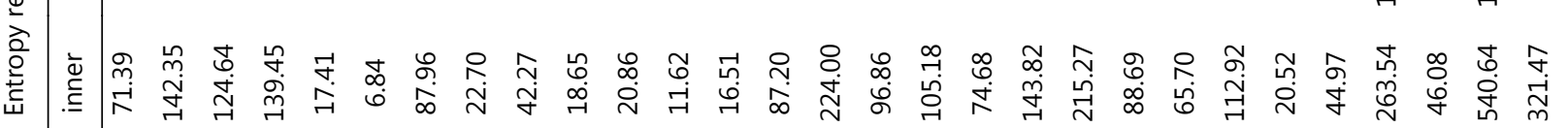

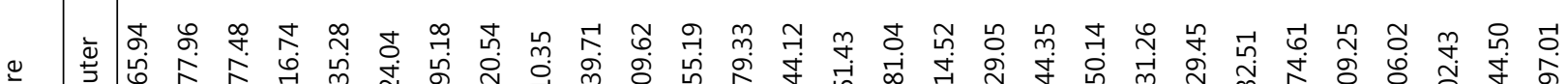
氙 可

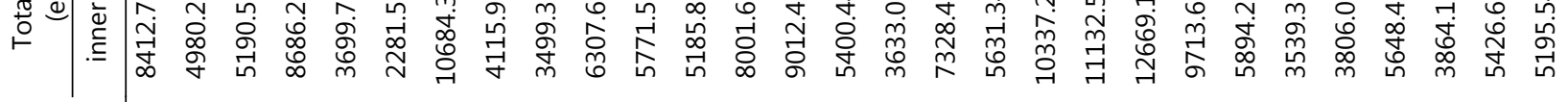

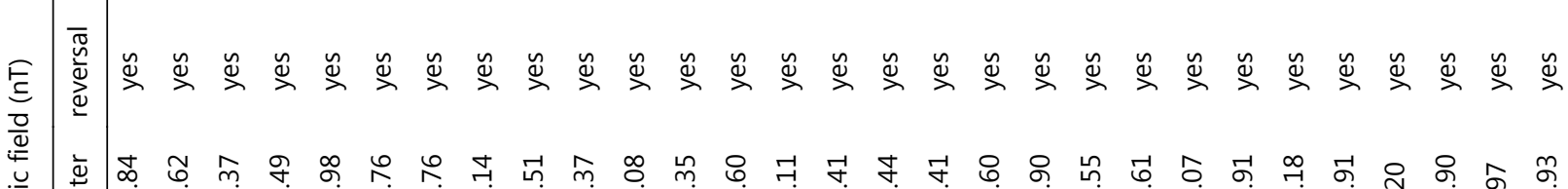
馬

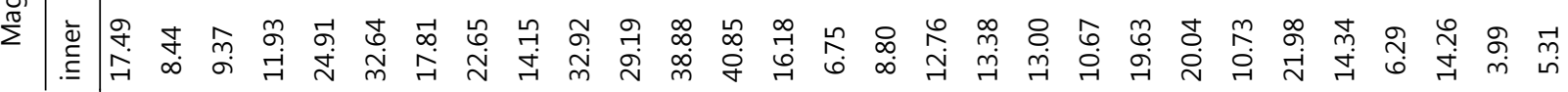

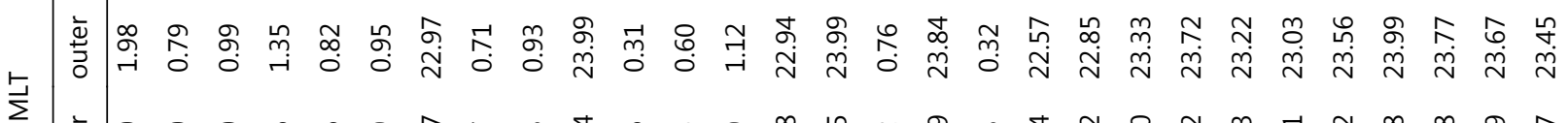

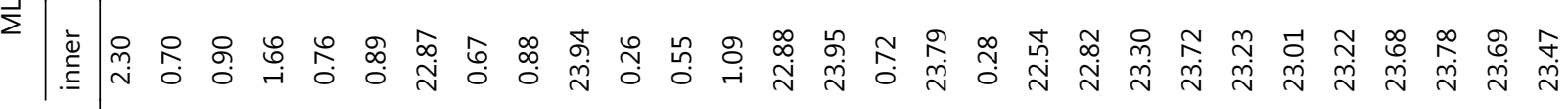
逐出

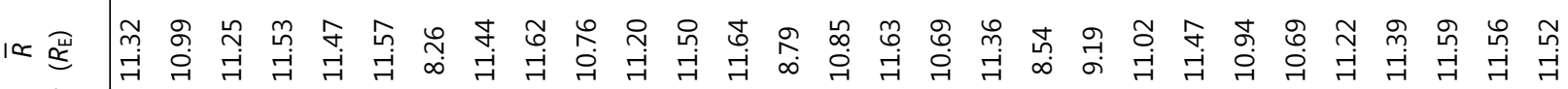

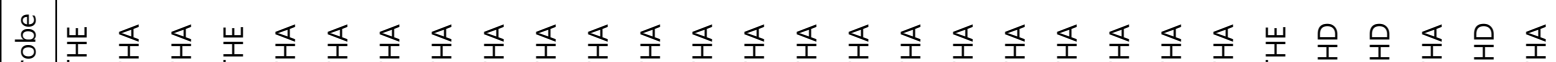
«) ङ 龸

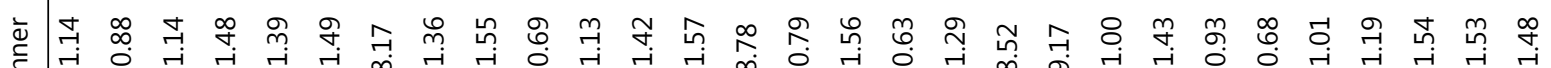

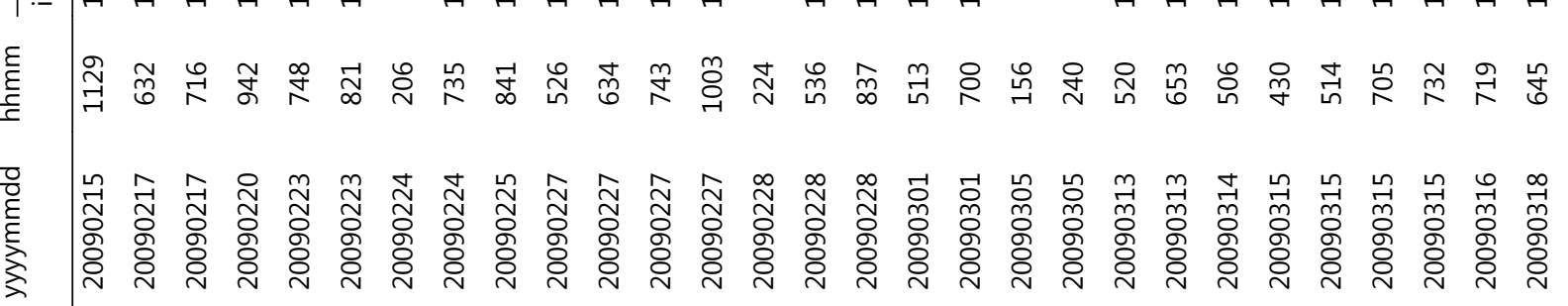




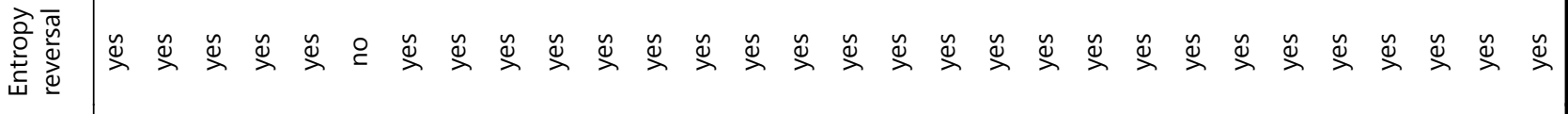

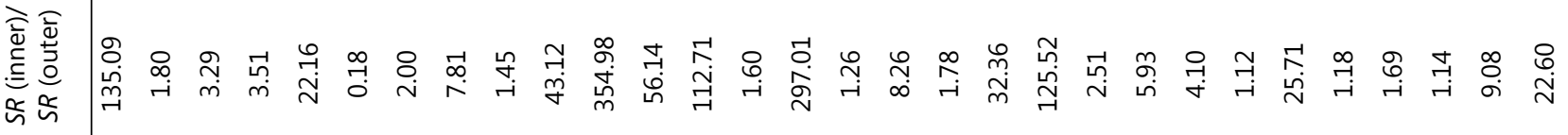

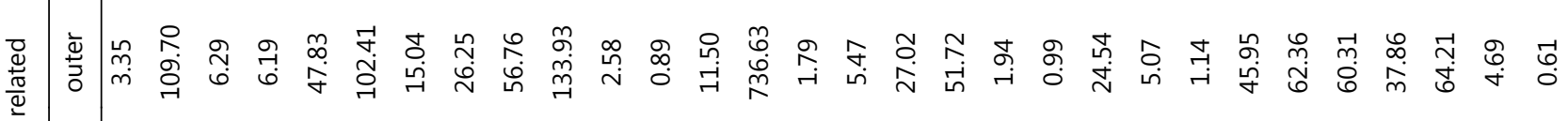

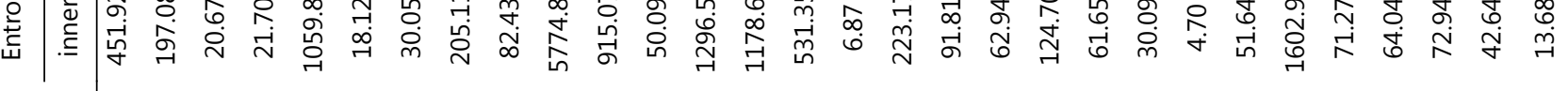

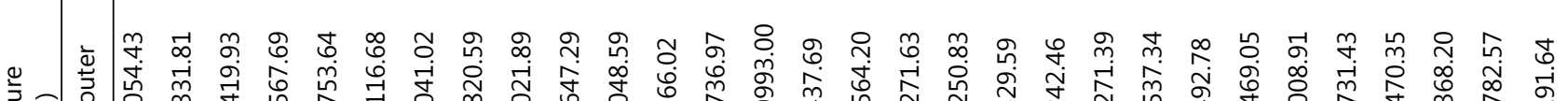

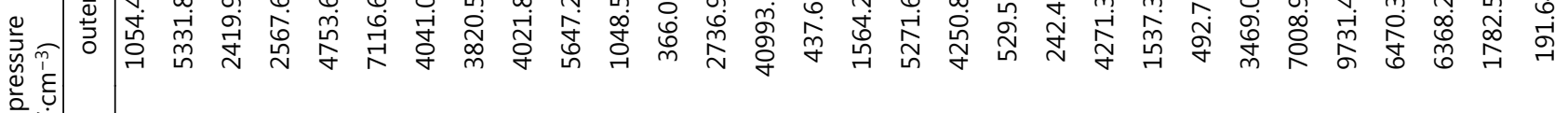

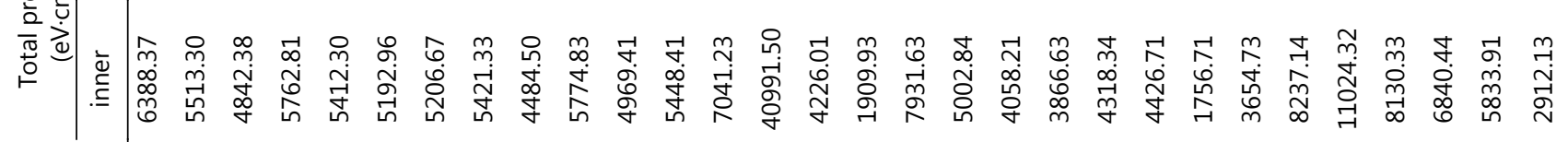

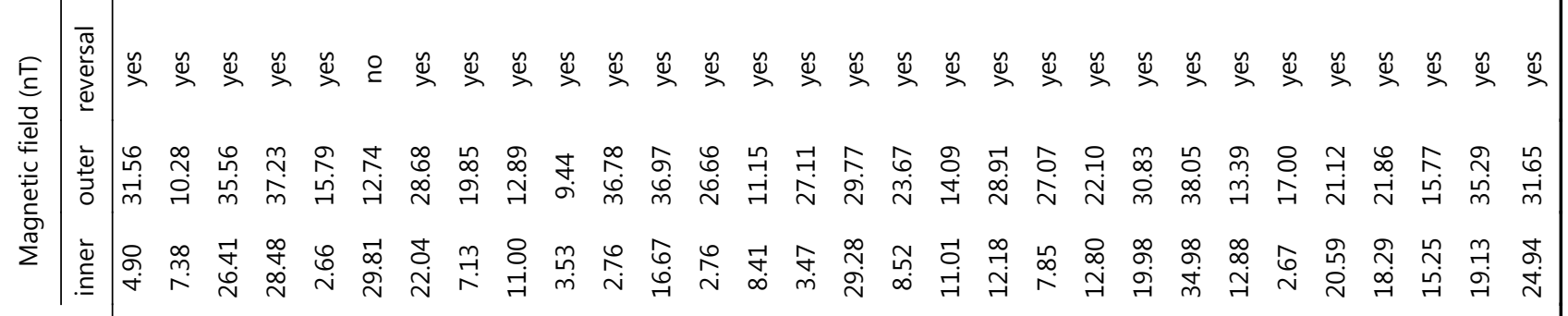

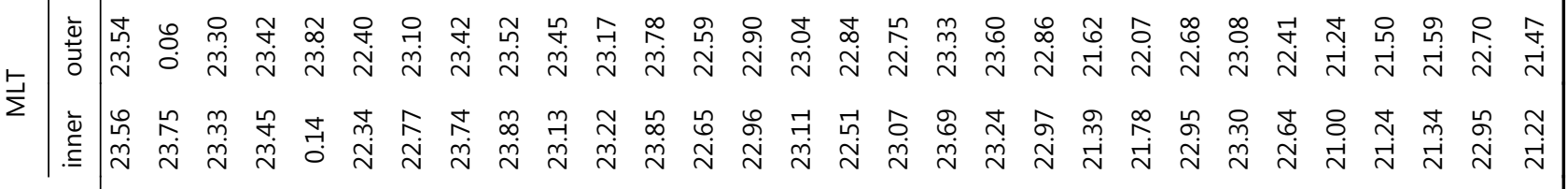

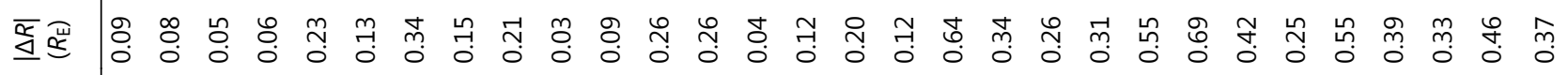
๙

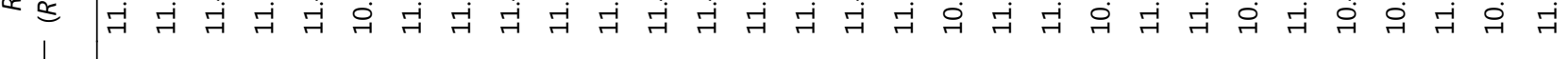

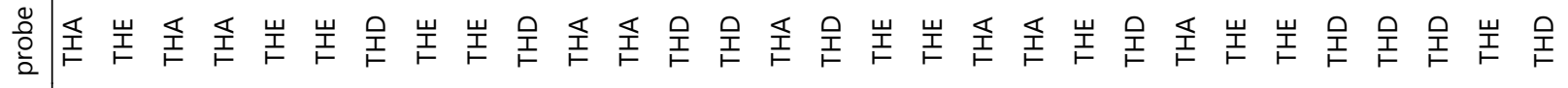

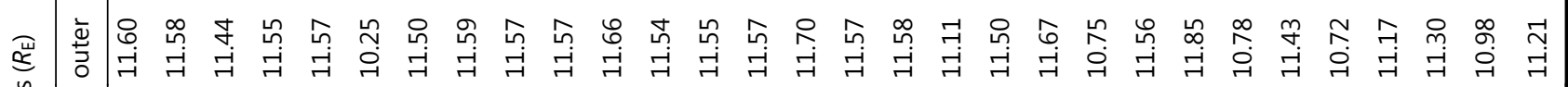

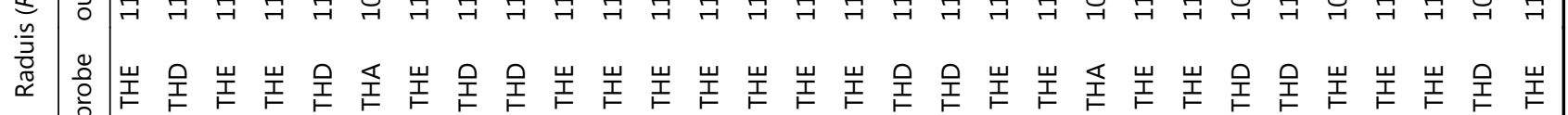

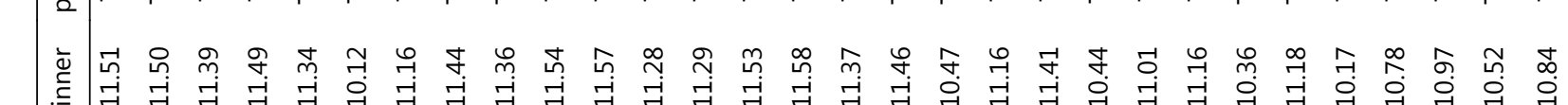

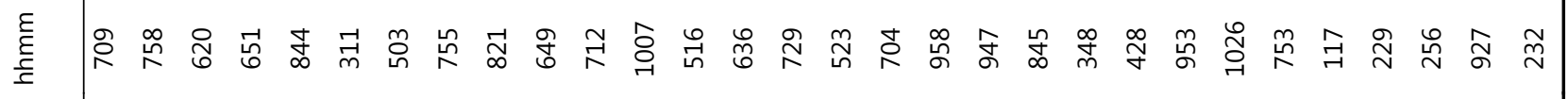

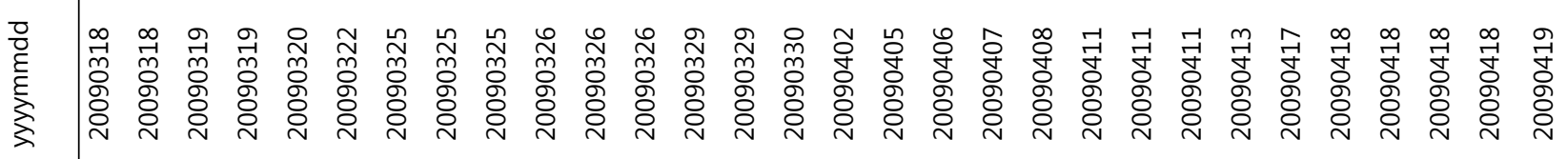

